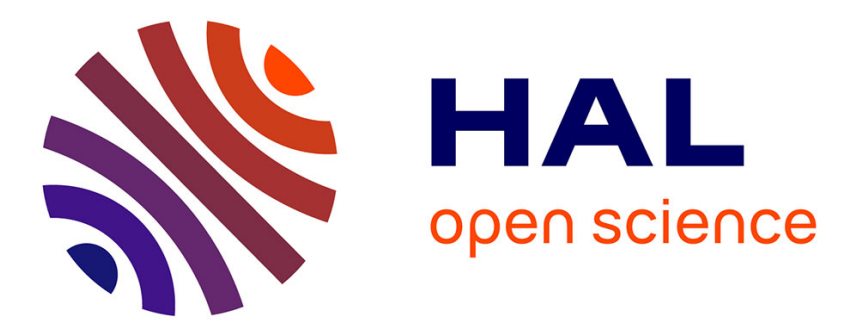

\title{
A numerical algorithm coupling a bifurcating indicator and a direct method for the computation of Hopf bifurcation points in fluid mechanics
}

\author{
Anthony Brézillon, Grégory Girault, Jean-Marc Cadou
}

\section{- To cite this version:}

Anthony Brézillon, Grégory Girault, Jean-Marc Cadou. A numerical algorithm coupling a bifurcating indicator and a direct method for the computation of Hopf bifurcation points in fluid mechanics. Computers and Fluids, 2010, 39 (7), pp.1226-1240. 10.1016/j.compfluid.2010.03.006 . hal-00493902

\section{HAL Id: hal-00493902 \\ https://hal.science/hal-00493902}

Submitted on 21 Jun 2018

HAL is a multi-disciplinary open access archive for the deposit and dissemination of scientific research documents, whether they are published or not. The documents may come from teaching and research institutions in France or abroad, or from public or private research centers.
L'archive ouverte pluridisciplinaire HAL, est destinée au dépôt et à la diffusion de documents scientifiques de niveau recherche, publiés ou non, émanant des établissements d'enseignement et de recherche français ou étrangers, des laboratoires publics ou privés. 


\title{
A numerical algorithm coupling a bifurcating indicator and a direct method for the computation of Hopf bifurcation points in fluid mechanics
}

\author{
A. Brezillon ${ }^{\mathrm{a}}$, G. Girault ${ }^{\mathrm{b}}$, J.M. Cadou ${ }^{\mathrm{a}, *}$

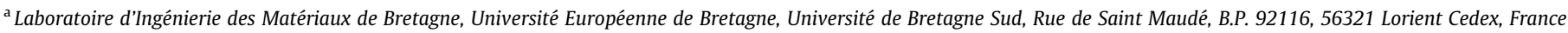 \\ ${ }^{\mathrm{b}}$ Centre de Recherche des Écoles de Saint-Cyr Coëtquidan, Écoles de Coëtquidan, 56381 Guer Cedex, France
}

\begin{abstract}
This paper deals with the computation of Hopf bifurcation points in fluid mechanics. This computation is done by coupling a bifurcation indicator proposed recently (Cadou et al., 2006) [1] and a direct method (Jackson, 1987; Jepson, 1981) [2,3] which consists in solving an augmented system whose solutions are Hopf bifurcation points. The bifurcation indicator gives initial critical values (Reynolds number, Strouhal frequency) for the direct method iterations. Some classical numerical examples from fluid mechanics, in two dimensions, are studied to demonstrate the efficiency and the reliability of such an algorithm.
\end{abstract}

\section{Introduction}

In fluid mechanics, flow can change when the Reynolds number reaches critical values. In this paper, we focus on the transition between a steady flow to a time-periodic solution. This modification characterizes an instability which is called Hopf's bifurcation. The present study concerns the numerical computations of these bifurcation points. However, to be precise, we do not discuss, in this paper, the stability of the flow after these instabilities. With the numerical methods presented in this paper, it is then impossible to give information on the kind of instability (subcritical or supercritical bifurcation for example) found. From a mathematical point of view, Hopf bifurcations correspond to two conjugated complex eigenvalues of the Jacobian matrix crossing the imaginary axis. The method to compute numerically this kind of instability can be classified into two families.

The first one is the family of indirect methods which consist in following a test function which has the property to be null in a Hopf bifurcation point $[1,4,5]$. This test function can be the smallest real part of one or more eigenvalues of the Jacobian matrix [6]. When this real part is equal to zero, then a Hopf bifurcation point is found. Nevertheless, as numerical fluid mechanics requires fine spatial discretization, the eigenvalue computation can lead to long CPU time. Moreover, due to non symmetric matrices, specific algorithms have to be used, for example the Arnoldi method [7].

\footnotetext{
* Corresponding author.

E-mail address: jean-marc.cadou@univ-ubs.fr (J.M. Cadou).
}

In a recent work [1], the authors proposed an indirect method based on a bifurcation indicator initially introduced in solid mechanics $[8,9]$. These indicators are scalar functions which have the property to be null in a bifurcation point. In Ref. [1], two indicators have been proposed. The first one allows for the characterization of stationary instabilities, such as the loss of symmetry in the flow and has been used to study the Coanda effect [10]. The second one leads to determining precisely Hopf bifurcation points. This indicator was first introduced in Refs. [11,12], respectively for models with small numbers of degrees of freedom and solid mechanics involving following loads. This bifurcation indicator is a function of the Reynolds number and the frequency. In Ref. [1], the bifurcation indicator is computed for a fixed value of the Reynolds number. It means that for a given value of the Reynolds number, the evolution of this indicator is evaluated versus the frequency. As the computation cost of this indicator can be great, an Asymptotic Numerical Method is used [13]. The latter permits the user to obtain analytic solutions. Nevertheless, with this method, the user has to follow the minimum value of the indicator versus the Reynolds and finally isolate the critical values of the flow indicating a Hopf bifurcation. This method is not an automatic ones and needs a great deal of post-analysis work for each computation. Moreover, precisely computing the critical values of the flow can lead to a great amount of indicator calculus and therefore to long CPU time.

The second family of methods used to characterize Hopf bifurcation points is the direct methods. They consist in the resolution of a nonlinear augmented system whose solutions are the Hopf 
bifurcation points. One can cite the methods proposed by Jepson [3] and Griewank and Reddien [14] which are solved by a Newton method [2]. These methods need initial values close to the bifurcation point to assure convergence. Usually $[2,15]$, initial values are obtained by the computation of some eigenvalues of the Jacobian matrix. In fact, one must isolate or at least have an idea of what the eigenvalues which can cross the imaginary axis are. Unfortunately, as this operator depends on the Reynolds number, the eigenvalues also do. One might note that their evolution with this number is not linear [16]. Consequently, it can be difficult to estimate precisely the eigenvalues which can cross the imaginary axis. Moreover, the numerical resolution of fluid mechanics requires a fine spatial discretization which can lead to an extensive matrix operator. The use of eigenvalues as initial values can then be quite difficult.

To avoid these eigenvalue computations, some authors [17] propose the quantities coming from an unsteady Navier-Stokes simulation as initial values. If for a given Reynolds number, the flow is time periodic, a Fourier transformation to the time series is applied to determine the critical frequency. Additional relations help give an approximation of the complex eigenvector [17].

In this work, we propose to use the quantities coming from the bifurcation indicator proposed in Ref. [1] as initial approximations for the augmented system proposed by Jepson [3] and Griewank and Reddien [14]. The basic idea is to associate the advantages of both methods to obtain a simple and efficient algorithm for the computation of Hopf bifurcation points. Indeed, the latter method can easily give good initial guesses but can demand long CPU time if precise values of the critical flow parameters are required. The direct method can compute precise critical numbers in few iterations if initial approximations are relatively close to the bifurcation point.

This paper is organized as follows. In the first part, the governing equations to be solved are recalled. The second part is concerned by the presentation of the two methods: firstly the direct method and secondly the bifurcation indicator. The numerical scheme is introduced during the third part. Finally, numerical examples, concerning classical tests in fluid mechanics are reported in the last part of this paper.

\section{Governing equations}

The Navier-Stokes equations for a Newtonian and incompressible fluid are the following:

$$
\left\{\begin{array}{l}
\frac{\partial u_{i}}{\partial t}-v u_{i, j j}+u_{j} u_{i, j}+\frac{1}{\rho} p_{, i}=0 \text { in } \Omega \\
u_{i, i}=0 \text { in } \Omega \\
u=\lambda u_{d} \text { on } \partial_{u} \Omega
\end{array}\right.
$$

where $u$ and $p$ are respectively the velocity and the pressure, $u_{d}$ is the imposed velocity in the boundary $\partial_{u} \Omega$ and $\rho$ is the density. In Eq. (1), $\lambda$ designates the intensity of the imposed velocity and can be identified by the Reynolds number, denoted Re, which is defined by using a reference length and the kinetic viscosity $v$. The previous equations are written in the following operator form:

$M(\dot{U})+L(U)+Q(U, U)-\lambda F=0$ in $\Omega$

where $M$ is the mass matrix, $L(U)$ and $Q(U, U)$ are respectively the linear and quadratic operators and $U$ is a mixed unknown vector defined by:

$U=\left\{\begin{array}{l}u \\ p\end{array}\right\}$

In Eq. (2), a load vector $F$ is introduced which is identical to the velocity imposed in the boundary $\partial_{u} \Omega$. For a more precise definition of these operators, the reader can refer to Ref. [18].
To study the stability of the Navier-Stokes equations (1), a perturbation vector $\mathscr{V}$ from the steady solution $U^{S}$ is introduced in Eq. (2) and by neglecting the second order terms in $\mathscr{V}$, the latter equation becomes:

$M(\dot{\mathscr{V}})+L(\mathscr{V})+Q\left(\mathscr{V}, U^{S}\right)+Q\left(U^{S}, \mathscr{V}\right)=0$ in $\Omega$

As $U^{S}$ is a steady solution of the Navier-Stokes equations, $U^{S}$ verifies:

$L\left(U^{S}\right)+Q\left(U^{S}, U^{S}\right)-\lambda^{S} F=0$ in $\Omega$

where $\lambda^{S}$ is equivalent to the Reynolds number at the steady solution $U^{S}$.

A Hopf bifurcation occurs when two eigenvalues of the Jacobian matrix cross the imaginary axis. So the velocity perturbation is sought under the form:

$\mathscr{V}(\mathbf{x}, \mathrm{t})=V(\mathbf{x}) \cdot e^{i \omega t}$

where $\omega$ is the frequency, $V$ the complex eigenvector and $\mathbf{x}=(x, y)$ is the position vector of any point in the flow domain $\Omega$. The previous expression is introduced into Eq. (4) which becomes, vector $\mathbf{x}$ being omitted for the sake of simplicity:

$\left\{\begin{array}{l}i \omega M(V)+L(V)+Q\left(V, U^{S}\right)+Q\left(U^{S}, V\right)=0 \text { in } \Omega \\ V_{u}=0 \text { on } \partial_{u} \Omega\end{array}\right.$

where $V_{u}$ designates the velocity part of the mixed unknown vector $V$. The latter is given by:

$V=V^{a}+i V^{b}$

In the previous expression, $V^{a}$ and $V^{b}$ are respectively the real and imaginary part of $V$. Finally, by introducing relation (8) into Eq. (7), a Hopf bifurcation point is the solution of the following system:

$$
\left\{\begin{array}{l}
L\left(U^{S}\right)+Q\left(U^{S}, U^{S}\right)-\lambda^{S} F=0 \text { in } \Omega \\
L\left(V^{a}\right)+Q\left(V^{a}, U^{S}\right)+Q\left(U^{S}, V^{a}\right)-\omega M\left(V^{b}\right)=0 \text { in } \Omega \\
L\left(V^{b}\right)+Q\left(V^{b}, U^{S}\right)+Q\left(U^{S}, V^{b}\right)+\omega M\left(V^{a}\right)=0 \text { in } \Omega \\
V_{u}^{a}=0 \text { on } \partial_{u} \Omega \\
V_{u}^{b}=0 \text { on } \partial_{u} \Omega
\end{array}\right.
$$

The previous nonlinear system is rewritten in the following condensed form:

$$
\left\{\begin{array}{l}
\mathscr{L}(\Lambda)=0 \text { in } \Omega \\
V_{u}^{a}=0 \text { on } \partial_{u} \Omega \\
V_{u}^{b}=0 \text { on } \partial_{u} \Omega
\end{array}\right.
$$

where the unknown $\Lambda$ is defined by $\Lambda={ }^{t}\left\{U^{S}, V^{a}, V^{b}, \lambda^{\mathrm{S}}, \omega\right\}$. Additional normalization conditions have to be added to the previous system to obtain a well-posed problem. These conditions are specified in the following sections where the methods used to compute the unknown vector $\Lambda$ are presented.

\section{Methods for computing Hopf bifurcation points}

To make this paper self-contained, let us recall the two methods used to compute bifurcation points. The coupling between these methods is also presented.

\subsection{Direct method}

The method proposed by Jepson [3] consists in solving problem (9) with the help of the Newton method. The unknown vector $\Lambda$ is then sought under the following expression:

$\Lambda=\Lambda_{0}+\Delta \Lambda$ 
where $\Lambda_{0}$ is a known initial approximation and $\Delta \Lambda$ is the unknown increment. The previous relation is introduced into Eq. (10) and by neglecting the second order terms in $\Delta \Lambda$, one obtains the equations to be solved at each iteration of the Newton method:

$$
\left\{\begin{array}{l}
\mathscr{L}_{t}^{\Lambda_{0}}(\Delta \Lambda)=\mathscr{R}^{\Lambda_{0}} \text { in } \Omega \\
\Delta V_{u}^{a}=0 \text { on } \partial_{u} \Omega \\
\Delta V_{u}^{b}=0 \text { on } \partial_{u} \Omega
\end{array}\right.
$$

where $\mathscr{L}_{t}^{\Lambda_{0}}(\Delta \Lambda)$ and $\mathscr{R}^{\Lambda_{0}}$ are respectively the tangent operator and the residual vector computed at the initial point $\Lambda_{0}$. These quantities are precisely defined in Appendix A.

To have a well-posed problem (25), two additional conditions are introduced $[2,3,14]$ :

$$
\left\{\begin{array}{l}
\left\langle l, V_{u}^{a}\right\rangle=0 \\
\left\langle l, V_{u}^{b}\right\rangle=1
\end{array}\right.
$$

where $l$ is a given vector (in our case an arbitrary chosen vector) and $\langle\bullet, \bullet\rangle$ stands for the euclidian scalar product. Although these normalization conditions can lead to turning points (i.e. $\omega=0$ and $\left.V^{a}=0\right)[16,17]$, they are linear expressions and their numerical treatment is easy to do. Discussions about such a choice are given in Refs. [3,17].

\subsection{Bifurcation indicator}

In Ref. [1], the authors have proposed an indicator, the property of which is to be null at a Hopf birfurcation point. Instead of solving the augmented system (9), they have fixed the Reynolds number (i.e. the steady solution $U^{S}$ is known) and they have computed the unknowns $\left(V^{a}, V^{b}, \omega\right)$ which satisfied system (9) with the help of an indicator. The steady solution is then computed independently by using an Asymptotic Numerical Method [18]. Eq. (9) are then replaced by the following ones:

$$
\left\{\begin{array}{l}
L\left(V^{a}\right)+Q\left(V^{a}, U^{S}\right)+Q\left(U^{S}, V^{a}\right)-\left(\omega_{0}+\hat{\omega}\right) M\left(V^{b}\right)=\Phi f \text { in } \Omega \\
L\left(V^{b}\right)+Q\left(V^{b}, U^{S}\right)+Q\left(U^{S}, V^{b}\right)+\left(\omega_{0}+\hat{\omega}\right) M\left(V^{a}\right)=0 \text { in } \Omega \\
V_{u}^{a}=0 \text { on } \partial_{u} \Omega \\
V_{u}^{b}=0 \text { on } \partial_{u} \Omega
\end{array}\right.
$$

where $f$ is a known random vector (chosen real), $\Phi$ is an unknown scalar, $\omega_{0}$ designates the initial angular frequency. In the numerical examples presented in this paper, $\omega_{0}$ is equal to zero. This is not a rule and $\omega_{0}$ can be chosen close to a critical value of the angular frequency.

The scalar $\Phi$ is the bifurcation indicator. Determining a Hopf bifurcation point consists in finding, for the steady solutions, the values $\omega=\omega_{0}+\hat{\omega}$ where $\Phi$ is equal to zero. The previous system can be solved by using a Newton method for each value of the angular frequency $\omega$. Nevertheless as the resolution of system (14) requires the triangulation of a great size operator, the total CPU time for the computation of $\Phi$ can be great. To avoid this, a perturbation method is proposed to solve system (14). The unknown vector $\mathrm{X}=\left(\Phi, V^{a}, V^{b}\right)$ is then sought as an integro-power series with respect to the angular frequency $\hat{\omega}$ :

$\mathrm{X}=\mathrm{X}_{0}+\hat{\omega} \mathrm{X}_{1}+\hat{\omega}^{2} \mathrm{X}_{2}+\cdots+\hat{\omega}^{p} \mathrm{X}_{p}$

To obtain a well-formulated problem, the following addition condition is required:

$\left\|V_{u}\right\|^{2}=\left\|W_{u_{0}}\right\|^{2}$

where $\|\bullet\|$ designates the euclidian norm of the vector ' $\bullet$ '. The additional condition (16) is chosen to avoid numerical instabilities (see Ref. [1] for more details). The vector $W_{u_{0}}$ is the solution to problem
(14) where $\omega=0$ and $\Phi_{0}$ equal to 1 . The asymptotic expansions (15) are then inserted in Eq. (14) and with balancing terms with identical powers of $\hat{\omega}$, we obtain the following set of linear equations:

\section{Order 0 in $\hat{\omega}$}

$$
\left\{\begin{array}{l}
L_{t}\left(V_{0}^{a}\right)-\omega_{0} M\left(V_{0}^{b}\right)=\Phi_{0} f \text { in } \Omega \\
L_{t}\left(V_{0}^{b}\right)+\omega_{0} M\left(V_{0}^{a}\right)=0 \text { in } \Omega \\
V_{u_{0}}^{a}=0 \text { on } \partial_{u} \Omega \\
V_{u_{0}}^{b}=0 \text { on } \partial_{u} \Omega \\
\left\|V_{u_{0}}\right\|^{2}=\left\|W_{u_{0}}\right\|^{2}
\end{array}\right.
$$

\section{Order $\mathbf{p}$ in $\hat{\omega}$}

$$
\left\{\begin{array}{l}
L_{t}\left(V_{p}^{a}\right)-\omega_{0} M\left(V_{p}^{b}\right)=\Phi_{p} f+M\left(V_{p-1}^{b}\right) \text { in } \Omega \\
L_{t}\left(V_{p}^{b}\right)+\omega_{0} M\left(V_{p}^{a}\right)=-M\left(V_{p-1}^{a}\right) \text { in } \Omega \\
V_{u_{p}}^{a}=0 \text { on } \partial_{u} \Omega \\
V_{u_{p}}^{b}=0 \text { on } \partial_{u} \Omega \\
\text { Forp }=1, \quad\left\langle V_{u_{1}}, V_{u_{0}}\right\rangle=0 \\
\text { Forp } \geqslant 2, \quad\left\langle V_{u_{p}}, V_{u_{0}}\right\rangle+\sum_{r=1}^{p-1}\left\langle V_{u_{(p-r)}}, V_{u_{r}}\right\rangle=0
\end{array}\right.
$$

The linear sytems (17) and (18) all have the same operator. So only one triangulation and $(p+1)$ foreward/backward substitutions permit the computation of the solution (15). In the previous systems, the tangent operator $L_{t}$ is the one which is defined in Eq. (27) in Appendix A. The validity range of these asymptotic expansions, which means the maximum value of $\hat{\omega}$, is determined by a simple criterion introduced in Ref. [13] and recalled in Appendix B.

From this maximum value of $\hat{\omega}$, one can compute a new initial value $\omega_{0}$ and by applying this scheme in a step-by-step manner, the indicator $\Phi$ can be determined for any value of $\omega$. To increase the validity range of asymptotic expansions and then decrease the number of operator triangulations, the polynomial expansions (15) are replaced by equivalent rational ones, called Padé approximants [19]:

$\mathrm{X}_{\text {Padé,p }}(\hat{\omega})-\mathrm{X}_{0}=\sum_{k=1}^{p-1} \frac{P_{(p-1-k)}(\hat{\omega})}{Q_{(p-1)}(\hat{\omega})} \hat{\omega}^{k} \mathrm{X}_{k}$

where $P_{k}$ and $Q_{k}$ are polynoms of degree $k$. One can remark that all the fractions in (19) have the same denominator $Q_{k}$. This representation has been tested and evaluated in Refs. [20,21] and is generally preferred to limit the number of roots of the Padés denominator, the so-called "poles" of Padé approximants.

To compute the entire response indicator versus frequency, denoted by $\mathscr{G}_{\mathrm{Re}}(\Phi, \omega)$, a continuation method based on the Padé approximants and first proposed by Elhage-Hussein et al. [22] is used. As for the polynomial expansions, the computation of the validity range of these rational fractions is recalled in Appendix B. The latter is the only difference between the method proposed in Ref. [1] and the indicator used in this work. Numerical comparisons between these two representations are done in the first part of the numerical section (see Section 5.2).

With the previous relations, the curves $\mathscr{G}_{\mathrm{Re}}(\Phi, \omega)$ are computed for a fixed value of the Reynolds number. The determination of Hopf bifurcation points consists in finding where $\omega$ leads to a null value of the scalar $\Phi$ on these curves. In practice, it consists in 
following the minimas of the function $\mathscr{G}_{\mathrm{Re}}(\Phi, \omega)$ and then estimates the critical numbers of the flow where a Hopf bifurcation appears.

\subsection{The proposed algorithm}

The computation of a Hopf bifurcation point with the presented direct method in Section 3.1, requires an initial approximation of $\Lambda_{0}$ which is relatively close to the solution to ensure the convergence of the Newton method. The bifurcation indicator, introduced in the previous section, allows one to obtain an estimation of the critical values of the flow. Thus we propose the following algorithm for the computation of the Hopf bifurcating point:

0 . Set the parameters of the method:

(a) The area of interest for the Reynolds number: $\operatorname{Re} \in\left[\operatorname{Re}^{1}\right.$, $\left.\operatorname{Re}^{2}\right]$.

(b) The order of truncation of the asymptotic expansions: $p$ (expression (15)). In practice $p$ is between 15 and 20 .

(c) The number of steps, ns, in the continuation technique [22] (the range of interest for the perturbation parameter $\hat{\omega})$.

(d) The maximum number of iterations for the Newton method (relations (25)).

1. Compute the steady solution $U_{0}^{S}$ and the corresponding Reynolds number, Re. In this work, it is done with the help of ANM [18].

2. If $\operatorname{Re} \in\left[\operatorname{Re}^{1}, \operatorname{Re}^{2}\right]$, then:

- Indicator phase, for step $j=1, \ldots$, ns.

2.1 Computation of the $\Phi_{p}^{j}$ and $V_{p}^{a^{j}}$ and $V_{p}^{b^{j}}$ with the expressions (18).

2.2 Evaluation of the validity range of the Padé approximants with the criterion defined in Ref. [22] and Appendix B. This means computing the maximum value of the perturbation parameter $\hat{\omega}$, denoted $\hat{\omega}_{\text {max }}$.

2.3 Build the Padé approximants with the expressions (19) and $\hat{\omega}_{\max }$.

2.4 Continuation technique: $\omega_{0}^{j}=\omega_{0}^{j-1}+\hat{\omega}_{\max }, V_{0}^{a^{j}}=V_{\text {Padé }}^{a}(\hat{\omega}$ $\max ), V_{0}^{b^{j}}=V_{\text {Padé }}^{b}\left(\hat{\omega}_{\max }\right)$.

- Direct method phase

3.1 Iterations of the Newton method (relations (25) and (13)), with $U_{0}^{S}, V_{0}^{a}, V_{0}^{b}$ and $\omega_{0}$ coming from steps 2.1 to 2.5 until convergence or the maximum number of iterations reached.

To summarize, the bifurcation indicator gives an initial approximation $\Lambda_{0}$ to the direct method during steps 2.1-2.5 of the previous algorithm. We will show in the numerical section how these initial values can be chosen in the best manner.

\section{Spatial discretization}

Spatial discretization of the previous equations are performed by using the classical finite element method. The chosen finite element is a quadrilateral element, with 9 nodes for the velocity (biquadratic interpolation) and 3 for the pressure (linear interpolation) [23]. The continuity equation is solved by using a penalty method [23]. In the following and for the sake of simplicity, discrete and continuous quantities have the same names.

The finite element formulation applied to expressions (12) and (13) leads to a linear system with a matrix which has a size $\left(\mathbb{R}^{3 n+2} \times \mathbb{R}^{3 n+2}\right.$ ) (where $\mathrm{n}$ is the number of degrees of freedom). The triangulation of such an operator leads to a very long computational times. Therefore to avoid a large amount of computational time, the system (12) is solved in a two-step method [2]. Firstly, the stationary solution, $\Delta U^{S}$, is looked for under the following form:

$\left\{\begin{array}{l}\Delta U^{S}=\alpha+\Delta \lambda \beta \\ \text { with } \alpha=K_{t}^{-1} \cdot R^{S} \quad \text { and } \beta=K_{t}^{-1} \cdot F\end{array}\right.$

The previous relation is the discrete form of the linearized stationary Navier-Stokes Eq. (5) and $K_{t}$ is the corresponding tangent matrix. Let us precise that this tangent matrix is the discrete form of the tangent operator defined in Eq. (27) in Appendix A.

Secondly, vectors $\alpha$ and $\beta$ are substituted in the discrete expression of Eq. (12) which can be written:

$\left[\mathscr{K}_{t}\left(\Lambda_{0}\right)\right]\{\Delta \Gamma\}=\left\{\mathscr{R}^{\Lambda_{0}}\right\}$

where $\left[\mathscr{K}_{t}\left(\Lambda_{0}\right)\right],\{\Delta \Gamma\}$ and $\left\{\mathscr{R}^{\Lambda_{0}}\right\}$ represent respectively the tangent matrix, the unknown vector and the residual computed at the initial value $\Lambda_{0}$. The tangent matrix $\left[\mathscr{K}_{t}\left(\Lambda_{0}\right)\right]$ is approximatively the discrete form of the linear sytem (25) defined in Appendix A. In fact, the first equation (the stationary part) in Eq. (25) is removed and the stationary unknown, $\Delta U^{\mathrm{S}}$, is modified according to the expression (20).

The unknown vector $\{\Delta \Gamma\}$ is defined by the following expression:

$\{\Delta \Gamma\}={ }^{t}\left\{\Delta \lambda, \Delta V^{a}, \Delta V^{b}, \Delta \omega\right\}$

The computation of the vector $\{\Delta \Gamma\}$ is performed by the factorization of the operator $\left[\mathscr{K}_{t}\left(\Lambda_{0}\right)\right]$. As the size of this operator is $\left(\mathbb{R}^{2 n+2} \times \mathbb{R}^{2 n+2}\right)$, the computation cost is cheaper than the initial ones. Finally, the stationary part, $\Delta U^{\mathrm{S}}$, of the vector $\Delta \Lambda$ is determined by introducing $\Delta \lambda$ in the Eq. (20).

For the bifurcation indicator, the discrete linear system at the truncature order $p(18)$ is written:

$$
\left[\begin{array}{cc}
K_{t} & -\omega_{0} M \\
\omega_{0} M & K_{t}
\end{array}\right]\left\{\begin{array}{c}
V_{p}^{a} \\
V_{p}^{b}
\end{array}\right\}=\left\{\begin{array}{c}
\Phi_{p} f+M V_{p-1}^{b} \\
-M V_{p-1}^{a}
\end{array}\right\}
$$

where $M$ is the discrete mass matrix. Solving system (23), associated with the additional condition (16) written at the order $p$, gives all the unknowns $\left(V_{p}^{a}, V_{p}^{b}, \Phi_{p}\right)$. For all these linear systems, only one matrix triangulation is needed and ' $p$ ' backward and forward substitutions. As the size of this matrix is approximatively the same as that of the Newton method, this means that the computation of one step of the indicator requires the same computational times as a single iteration of the direct method. By using relation (19), one can build an analytical part of the curves $\mathscr{G}_{\operatorname{Re}}(\Phi, \omega)$. The entire curves, $\mathscr{G}_{\mathrm{Re}}(\Phi, \omega)$, are computed by using a continuation method based on the Padé approximants [22].

\section{Numerical results}

\subsection{Numerical examples}

The numerical tests presented in this paper are classical ones but have the advantage of presenting Hopf bifurcations which are relatively well-known. Hence, one considers two examples. The first one is the flow around a cylinder. This example is the same as in Ref. [2] excepted for the boundary conditions. Indeed, in this work, the outflow condition is a Dirichlet condition whereas in Ref. [2] a stress boundary condition is imposed. In fact, this Dirichlet condition can lead to spurious oscillations of the solutions. Nevertheless, as the considered Reynolds are relatively small (between 23 and 54), these spurious oscillations have not been observed in all our numerical computations. Let us remark that for higher Reynolds numbers, a stress boudary condition has to be imposed in the outflow. The second one is the lid-driven cavity. For this problem, several geometrical cases or aspect ratios $A$ are studied in this paper (see Table 1). 
Table 1

Mesh sizes used for the numerical tests.

\begin{tabular}{lll}
\hline Example & Aspect ratio & Number of d.o.f. \\
\hline Flow around a cylinder & - & 6112 \\
Lid-driven cavity & $A=0.8$ & 20.402 \\
& $A=1.0$ & 13.122 \\
& $A=1.5$ & 13.122 \\
\hline
\end{tabular}

The geometrical description of these examples and the boundary conditions are shown in Figs. 1 and 2 respectively for the flow around a cylinder and the lid-driven cavity. As discussed previously, the finite element used in the numerical tests is a quadrangle with nine velocity nodes and three for the pressure. The number of d.o.f. for the numerical tests is given in Table 1 .

We give in Tables 2 and 3 the critical Reynolds and Strouhal numbers reported by various researchers, respectivevly for the flow around a cylinder and the lid-driven cavity with an aspect ratio $A$ equal to 1 . We also indicate in these tables, for all the references, the numerical method used to characterize a Hopf bifurcation point. One can remark in these tables that, most of the works are based on an eigenvalue computation (EC in the Tables 2 and 3 ). In the latter and concerning the eigenvalue computation we do not distinguish the kind of eigensolution method used in the different references. For example, in Ref. [28] a subspace iteration is used, in Ref. [25] it is a classical QR algorithm

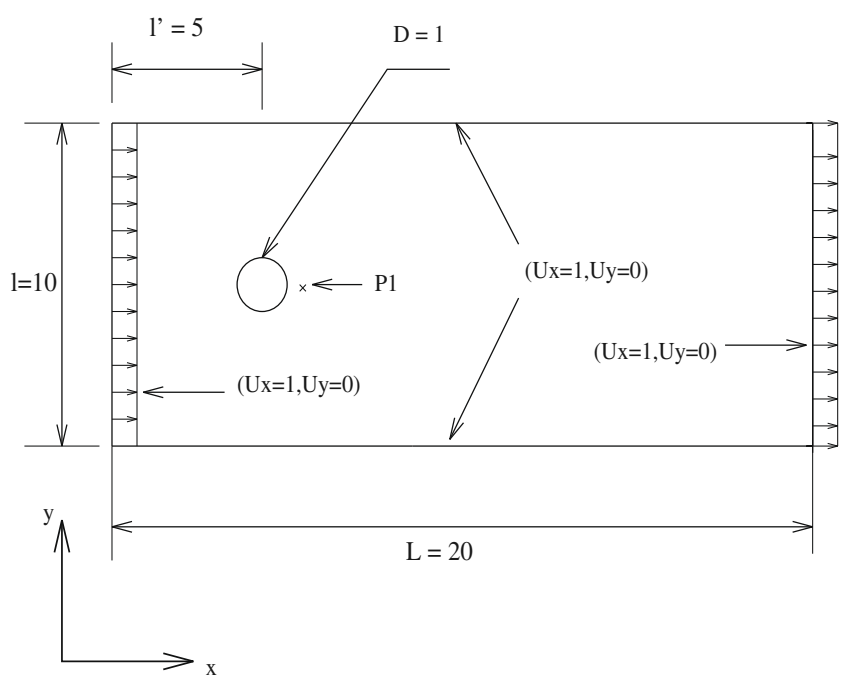

Fig. 1. Flow around a cylinder, description of the geometry.

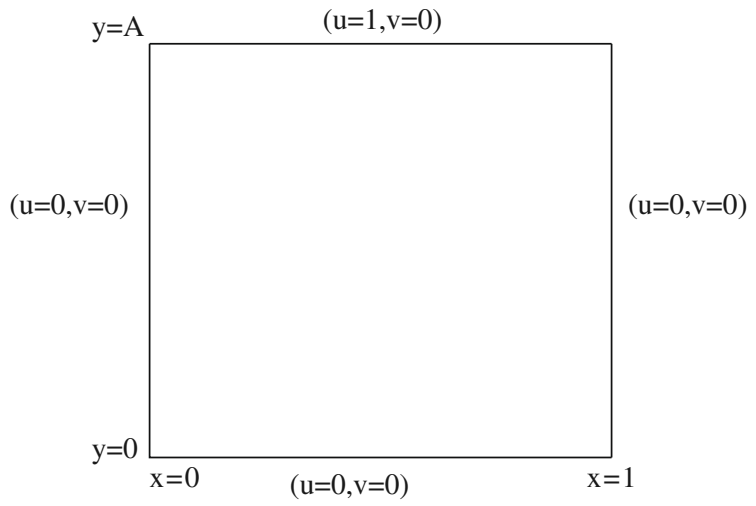

Fig. 2. Geometry for the 2-D lid-driven cavity flow.
Table 2

Comparison of the critical parameters for the first Hopf bifurcation from the literature. The following acronyms EC, DNS, NM respectively stand for eigenvalue computation, direct numerical simulations and Newton method. The considered example is the flow around a cylinder.

\begin{tabular}{llll}
\hline Authors & $\mathrm{Re}_{c}$ & $\mathrm{St}_{c}$ & Method \\
\hline Jackson [2] & 46.184 & 0.137 & $\mathrm{NM}$ \\
Cossu and Morino [25] & 45.63 & 0.112 & EC \\
Kumar and Mittal [26] & 46.88 & 0.116 & DNS and EC \\
Ding and Kawahara [27] & 46.389 & 0.126 & EC \\
Morzynski et al. [28] & 47 & 0.132 & EC \\
This study & 45.98 & 0.137 & \\
\hline
\end{tabular}

Table 3

Comparison of the critical parameters for the first Hopf bifurcation from the literature. The following acronyms EC, POD, DNS, NM, LE respectively stand for eigenvalue computation, proper orthogonal decomposition (associated to the computation of the eigenvalues of the reduced system), direct numerical simulations, Newton method, Lyapunov exponent. The considered example is the lid-driven cavity with $A=1$.

\begin{tabular}{llll}
\hline Authors & $\mathrm{Re}_{c}$ & $\mathrm{St}_{c}$ & Method \\
\hline Fortin et al. [5] & 8000 & 0.45 & $\mathrm{EC}$ \\
Boppana and Gajjar [6] & 8026.6 & 0.4497 & $\mathrm{EC}$ \\
Polishenko and Aidun [17] & 7763.4 & 0.45 & $\mathrm{NM}$ \\
Bruneau and Saad [29] & $8000-8050$ & - & $\mathrm{LE}$ \\
Peng et al. [30] & 7402 & 0.59 & $\mathrm{DNS}$ \\
Abouhamza and Pierre [31] & 8004.5 & - & $\mathrm{EC}$ \\
Gervais et al. [32] & 7960 & 0.45 & $\mathrm{EC}$ \\
& 8040 & 0.45 & $\mathrm{EC}$ \\
Tiesinga et al. [33] & 8375 & 0.4399 & $\mathrm{EC}$ \\
Cazemier et al. [34] & 7819 & 0.61 & $\mathrm{POD}$ \\
& 7972 & 0.45 & $\mathrm{DNS}$ \\
Auteri et al. [35] & $\approx 8018$ & 0.4496 & $\mathrm{DNS}$ \\
This study & 7890 & 0.44 & \\
\hline
\end{tabular}

and in Ref. [6] the eigenvalue problems is solved with an Arnoldi package. In Ref. [34], the authors have also computed the eigensolutions of the linearized perturbed Navier-Stokes equations. Nevertheless, a low order model (POD) is used to reduce the size of the Jacobian matrix.

One should remark that in Ref. [2], an eigenvalue computation is also realized (inverse iteration method) to give an initial guess for the Newton method. In Ref. [17], the Hopf bifurcation point is also computed with the help of a Newton method, but this time, the starting values for the iterative method are obtained with a time dependant Navier-Stokes simulation. From these tables, one can note that in a few works, a direct numerical simulation of the time dependant Navier-Stokes equations has been done. This is mainly due to the fact that this kind of technique requires a great amount of CPU time to localize precisely the bifurcation point Finally, in Ref. [29], the stability of the flow is studied by computing the first Lyapunov exponent of the linearized problem.

For these two classical benchmarks in the numerical study of flow stability, the literature's results presented in Tables 2 and 3 show that all the studies give approximatively the same critical Reynolds and Strouhal numbers. Hence, for the flow around a cylinder, the Hopf bifurcation occurs for a Reynolds and a Strouhal number respectively between [45-47] and [0.112-0.137]. For the lid-driven cavity, the Hopf bifurcation point is found for a Reynolds number included in the range [7400-8400] and the corresponding critical frequency is close to 0.45 . In Ref. [34], the found critical Strouhal number is greater than the other works reported in Table 3. This difference is explained by the fact that the small dynamical system (from which an eigenvalue computation is done) is obtained with a time dependant simulation realized at a Reynolds number equal to 22,000 . 
In the following, we first propose to give some explanations on how the bifurcation indicator [1] works. Next, we compare the computing performances obtained with the first version of the indicator [1] and the one based on the Padé approximants. In the following section, we show how this indicator is linked to the Newton method (see Section 3.1) to give an efficient algorithm for the computation of Hopf bifurcation points. A section is also devoted to the computational times of the proposed method. The proposed algorithm is then applied to the lid-driven cavity with geometrical aspect ratio equal to 0.8 and 1.5. In the last part of this numerical section, we show that the coupled method can easily give some supplementary Hopf bifurcation points.

\subsection{Bifurcation indicator}

We first propose to give some explanations about the bifurcation indicator presented in Section 3.2 and in Ref. [1]. We then consider the flow around the cylinder. The critical numbers for this flow are $\operatorname{Re}_{c}=45.98, \omega_{c}=3.97$ [1] and the corresponding Strouhal

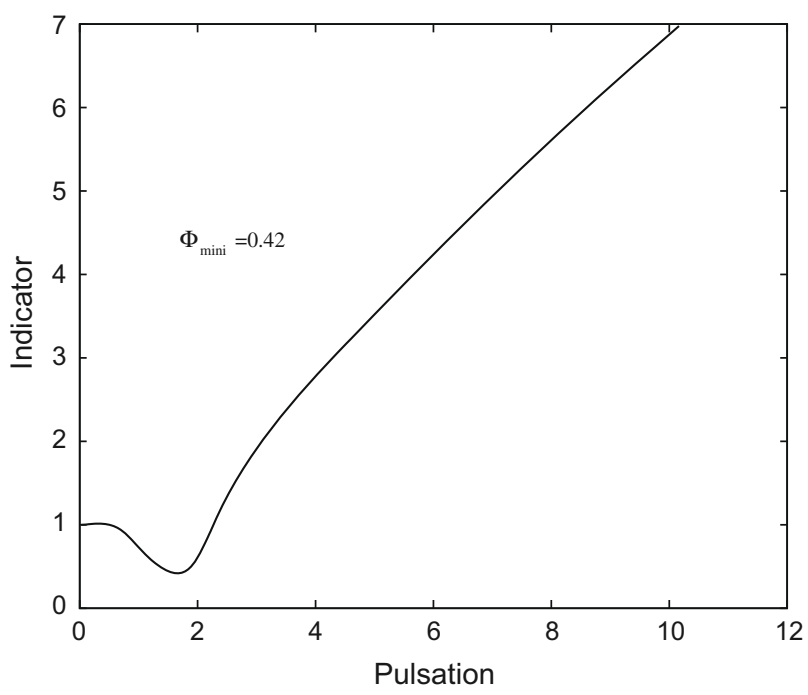

(a) $\operatorname{Re}=23.05$

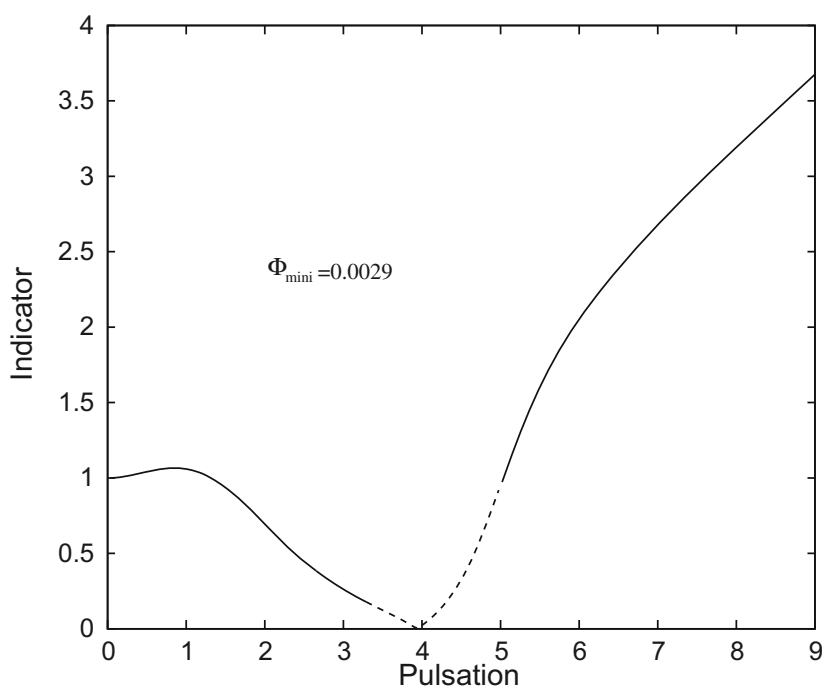

(c) $\operatorname{Re}=45.60$ number is equal to $\mathrm{St}_{c}=0.137$. Jackson [2] has found, for this example, the following values: $\operatorname{Re}_{c}=46.184$ and $\mathrm{St}_{c}=0.138$. The Strouhal number is computed from the following expression:

$\mathrm{St}=\frac{D \cdot \omega}{2 \pi u}$

where $u$ is the imposed velocity (see Fig. 1 ) and $D$ is the cylinder diameter. To understand how the indicator, $\Phi$, works we have computed it for a Reynolds number $\operatorname{Re} \in[20,60]$. The curves $\mathscr{G}_{\operatorname{Re}}(\Phi, \omega)$ for four values in the range of Reynolds numbers are shown in Fig. 3. We indicate for each curve, $\mathscr{G}_{\mathrm{Re}}(\Phi, \omega)$, the minimum value of the indicator $\Phi$. One can see in these curves that the minimum of the indicator decreases when we get closer to the critical Reynolds and increases when we get farther from this value. Indeed the minimum is equal to 0.42 for $\operatorname{Re}=23.05,0.19$ for $\operatorname{Re}=32.41$, 0.0029 for $\operatorname{Re}=45.60$ and 0.04 for $\operatorname{Re}=54.92$, which means that, according to the conclusions presented in Ref. [1] there is a Hopf bifurcation point between the Reynolds number 32.41 and 54.92. So to determine precisely this critical value one has to compute

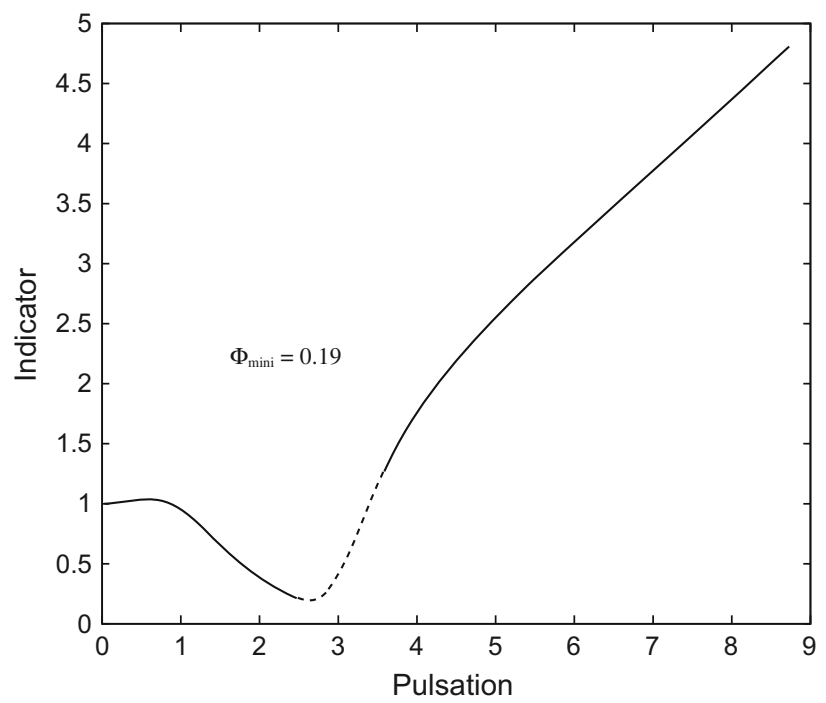

(b) $\operatorname{Re}=32.41$

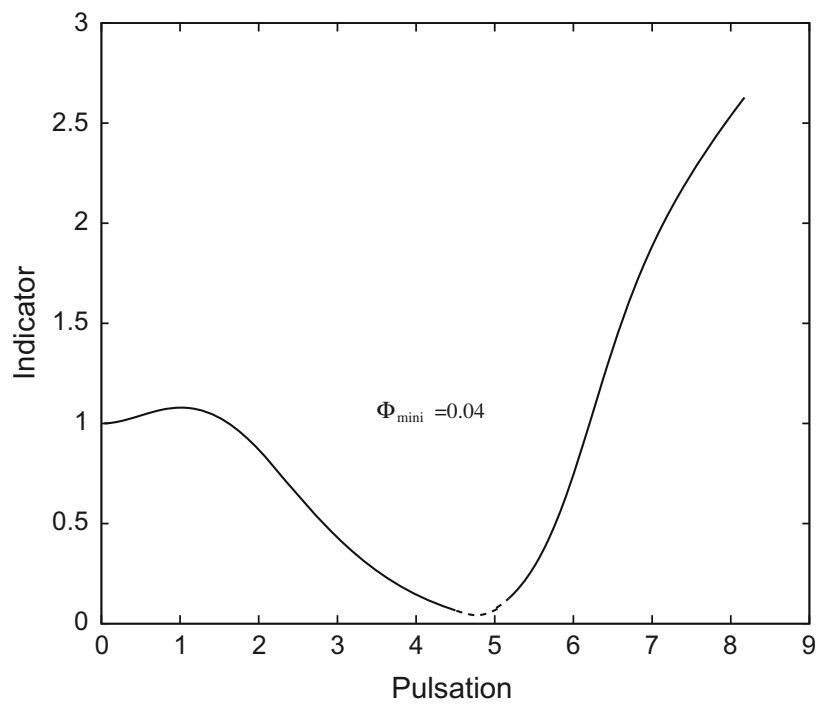

(d) $\operatorname{Re}=54.92$

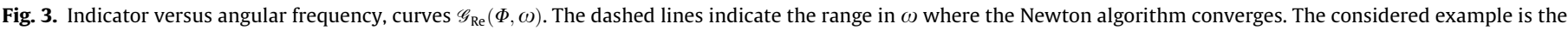
flow around a cylinder. 
supplementary curves $\mathscr{G}_{\mathrm{Re}}(\Phi, \omega)$ for some Reynolds numbers in this range. From these computations, one has to determine a new range of Reynolds number where the indicator is minimum and so on until this minimum reaches a fixed user given tolerance.

In Table 4, we give, for each Reynolds number considered in Fig. 3, the number of steps and the final value of the parameter $\omega$ reached. We consider the polynomial approximation (15) and the Padé approximants (19). The truncature order $p$ is fixed and equal to 20 according to the conclusions of previous studies about ANM [24]. In fact, in Table 4 we have fixed the number of steps of the Padé approximants to 20 and we give the number of steps demanded with the polynomial representation to reach the same final value of $\omega$. In this table, one can remark that the number of steps demanded with the Padé approximants is always smaller than with the polynomial representations.

To illustrate greater step lengths obtained with the Padé approximants, we have plotted in Figs. 4 and 5 the evolution of the indicator versus the angular frequency (for a Reynolds number equal to 39.01). In these figures, the square symbol indicates the starting point $\mathrm{X}_{0}$ of the asymptotic expansions (15). These starting points are determined by using expression (30). In these figures, the reference curve is the one obtained with the continuation method. In these curves, Fig. 5 being an enlarged view of Fig. 4, we have plotted for the second continuation point the evolutions

Table 4

Number of steps to compute the indicator by using the polynomial representation (15) or with the Padé approximants (19). The order of truncature $p$ is equal to 20 . The considered example is the flow around a cylinder.

\begin{tabular}{llll}
\hline & Re & Number of steps & $\omega$ reached \\
\hline Pade & 23.05 & 20 & 10.16 \\
Poly & & 26 & 10.17 \\
Pade & 32.41 & 20 & 8.71 \\
Poly & & 24 & 8.74 \\
Pade & 39.01 & 20 & 6.75 \\
Poly & & 23 & 7.21 \\
Pade & 45.60 & 20 & 4.00 \\
Poly & & 24 & 4.00 \\
Pade & 54.92 & 20 & 8.20 \\
Poly & & 23 & 8.23 \\
\hline
\end{tabular}

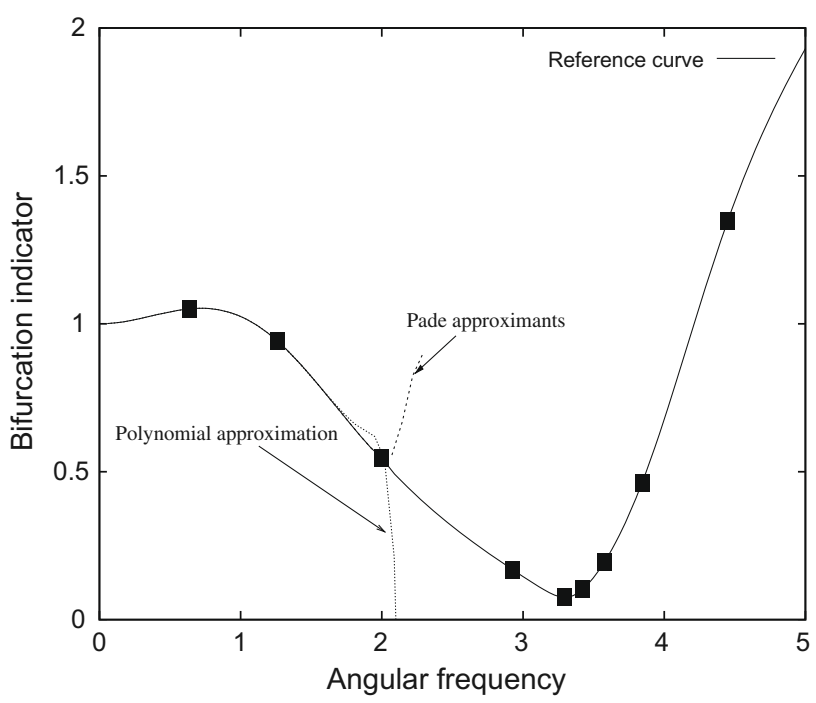

Fig. 4. Bifurcation indicator, comparison of the solutions obtained with Padé and polynomial approximation. $\mathrm{Re}=39.01$. Square symbols indicate the begining of the step. The considered example is the flow around a cylinder.

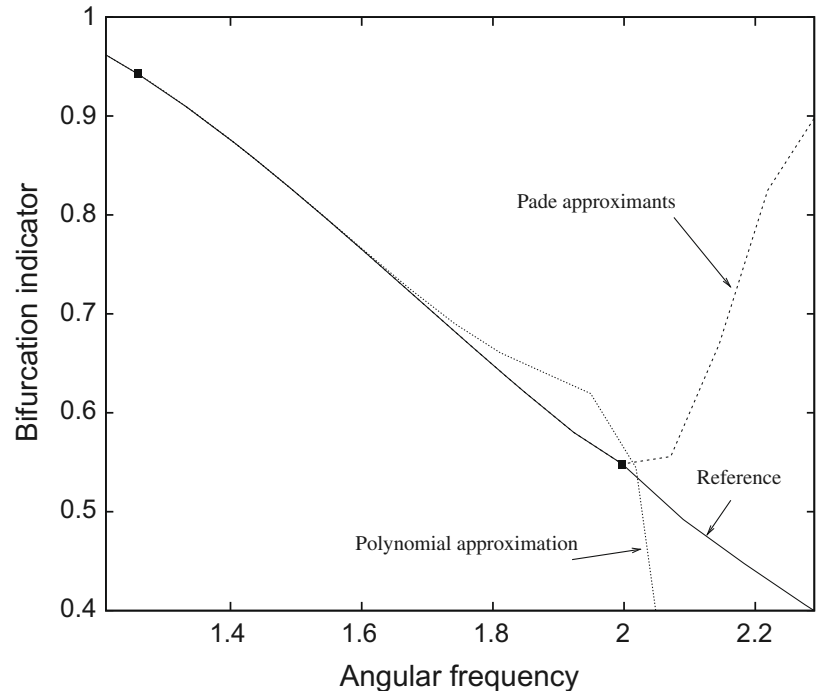

Fig. 5. Bifurcation indicator, comparison of the solutions obtained with Padé and polynomial approximation. $\mathrm{Re}=39.01$. Zoom of the Fig. 4 . The considered example is the flow around a cylinder.

of the polynomial approximations (15) and of the Padé approximants (19).

One can clearly see in these figures that the solution obtained with the Pade approximants is closer to the reference curve for greatest values of the angular frequency than with the polynomial approximations. It shows that the range of validity of the Padé approximants is generally greater than the one obtained with the polynomial approximations. Each step of the continuation method needs a single matrix triangulation (Eq. (23)) and $p$ backward/foreward substitution. The points where a matrix triangulation is realized are indicated by square symbols in Figs. 4 and 5 . We have reported in Table 5, the CPU time needed for the computation of one step of the indicator. One considers the CPU time for the matrix triangulation, for the computation of the solution of the $p$ linear systems (18) and for the construction of the Padé approximants (19). These times are given for the flow around a cylinder and the lid-driven cavity, respectively denoted by Examples 1 and 2 in Table 5. In the latter, one can see that the largest CPU time of one step of the perturbation method is for the matrix triangulation. The computation of all the unknows $\mathrm{X}_{p}$ requires approximatively $30 \%$ and $14 \%$ of the total CPU cost respectively for Examples 1 and 2. This short CPU time is due to the fact that the matrix triangulation is done only once, for the order of truncature equal to 0 , and for the supplementary unknowns (for $p \geqslant 1$ ), only forward and backard substitutions are required. Concerning the Padé approximants, Table 5 illustrates the fact that the CPU time for the construction of the rational fraction (19) is insignificant compared to what it needed for a matrix triangulation. Finally

Table 5

CPU time (in second) for one step of the indicator computation. The truncature order is equal to 20. Examples 1 and 2 designate respectively the flow around a cylinder and the 2-D lid-driven cavity. Computations are realized on a workstation DELL Precision 490.

\begin{tabular}{lcc}
\hline & $\begin{array}{l}\text { Example 1 (6112 } \\
\text { d.o.f.) }\end{array}$ & $\begin{array}{l}\text { Example 2 (13 122 } \\
\text { d.o.f.) }\end{array}$ \\
\hline Matrix triang & 7.3 & 58.67 \\
$\begin{array}{l}\text { Computations of all the } \\
X_{p}\end{array}$ & 2.83 & 9.64 \\
Padé approximants & 0.04 & 0.07 \\
Total & 10.13 & 68.31 \\
\hline
\end{tabular}


as the Pade approximants lead to larger step sizes than with the polynomial approximations, the CPU computing times to determine the indicator curves $\mathscr{G}_{\mathrm{Re}}(\Phi, \omega)$ is then reduced. This is an improvement of the bifurcation indicator compared to the ones previously presented in Ref. [1]. Comparisons of the computational cost between the indicator proposed in Ref. [1] and the method proposed in this work are realized in the following section.

Another important feature of the indicator curves is that, for a fixed number of steps, the maximum value of $\omega$ decreases when a bifurcating point is close. For example, in Table 4, one can see that for a Reynolds number equal to 23.05 with 20 steps and the Padé approximants, we get a maximum value of $\omega$ equal to 10.17, whereas for a Reynolds number equal to 45.60 , the curve $\mathscr{G}_{\mathrm{Re}}(\Phi, \omega)$ is computed only up to $\omega=4$. In fact, the nonlinear curve $\mathscr{G}_{\operatorname{Re}}(\Phi, \omega)$ is less regular when a bifurcation point is close to the considered Reynolds number. This is mainly due to the definition (16). Let us recall that this definition has been introduced in Ref. [1] to avoid numerical instabilities. In the following results, the Padé approximants are always the representation used for the computation of the curves $\mathscr{G}_{\operatorname{Re}}(\Phi, \omega)$.

We now propose to associate the direct method presented in Section 3.1 by taking the initial values from the curves $\mathscr{G}_{\operatorname{Re}}(\Phi, \omega)$.

\subsection{Results with the proposed algorithm}

We consider the same example as before (flow around a cylinder). For each curve $\mathscr{G}_{\mathrm{Re}}(\Phi, \omega)$ shown in Fig. 3, we choose initial values for the augmented system (12). It is quite easy to define these initial values. Indeed, as the perturbation method gives analytic solutions, one has to choose a value $\omega$ on the curves $\mathscr{G}_{\operatorname{Re}}(\Phi, \omega)$ and by introducing the chosen value in expressions (19), one obtains initial values for the Newton method. This has been done for the four value of the Reynolds number in the case of the flow around a cylinder. For a Reynolds number equal to 23.05 (Fig. 3a, no chosen value $\omega$ on this curve leads to a convergence of the Newton algorithm. For the other values of the Reynolds number, the area in $\omega$ for which the Newton method converges is plotted in dashed lines in Fig. 3. The closer the critical Reynolds number is, the wider the convergence area is.

In Fig. 6, we plot the number of necessary iterations to get the required accuracy with the Newton algorithm for the values of $\omega$

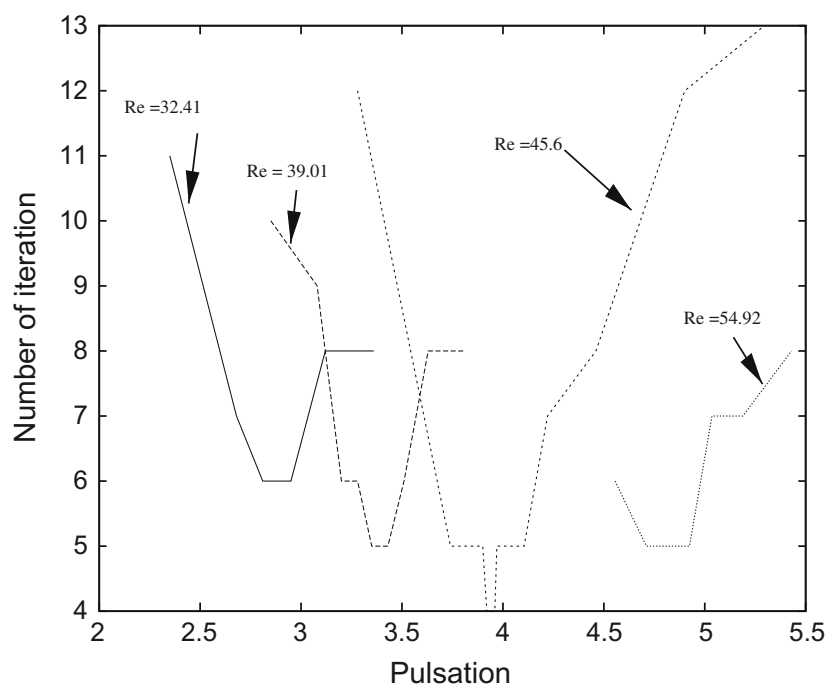

Fig. 6. Number of iterations of the Newton method (system (12)) versus pulsation for several Reynolds numbers. The convergence is reached when the following criteria are satisfied: $\left\|R^{S}\right\|<10^{-4},\left\|R^{a}\right\|<10^{-8}$ and $\left\|R^{b}\right\|<10^{-8}$. The considered example is the flow around a cylinder. highlighted in Fig. 3 by dashed lines. Obviously the minimum number of iterations is obtained when the Reynolds number is equal to 45.60. Nevertheless, one can remark that each curve $\mathscr{G}_{\mathrm{Re}}(\Phi, \omega)$ gives initial values leading to the convergence of the Newton method. Moreover, if on each curve $\mathscr{G}_{\operatorname{Re}}(\Phi, \omega)$, one chooses as an initial guess the value of $\omega$ for which the indicator $\Phi$ is minimum, the Newton method converges to the critical numbers of the flow which are $\mathrm{Re}_{c}=45.98$ and $\omega_{c}=3.972\left(\mathrm{St}_{c}=0.137\right)$. These critical values are very similar to those found in the literature and reported in Table 2.

We now consider the 2-D lid-driven cavity with an aspect ratio $A$ equal to 1 . We have computed the indicator curves, $\mathscr{G}_{\mathrm{Re}}(\Phi, \omega)$, for Reynolds numbers between 6000 and 11,000. The evolution of the indicator versus the Strouhal number for four values of the Reynolds number included in the studied range is plotted in Fig. 7. This example is interesting because the curves, $\mathscr{G}_{\mathrm{Re}}(\Phi, \mathrm{St})$, show a lot of minima of the indicator, $\Phi$, for $\mathrm{St}_{c}$ in $[0 ; 0.8]$. Moreover, these minima change with the Reynolds number. For example, when $\operatorname{Re}=7257$ the minimum of the indicator is reached for $\mathrm{St}_{c}$ close to 0.27 , whereas when $\mathrm{Re}=8089$ it is for $\mathrm{St}_{c}=0.44$. These values are summarized in Table 6 . For each minimum value of the curves $\mathscr{G}_{\mathrm{Re}}(\Phi, \mathrm{St})$, the direct method is used to determine precisely the critical values of the flow. The number of iterations of the Newton method and the found critical numbers are given in Table 6 .

For this example, the first Hopf bifurcation is found for a Reynolds number and a Strouhal number respectively equal to 7890 and 0.44 . These critical values are close to the ones found in the literature, see for example Table 3 or the Ref. [6] where a great number of works on this numerical test are summarized. To prove that our results correlate well with those of the literature, we plot in Fig. 8 the streamlines of the stationary solution (at $\operatorname{Re}=7890$, Fig. $8 \mathrm{a}$ ) and of the real and imaginary parts of the critical eigenvector (respectively Fig. $8 \mathrm{~b}$ and c). These figures are quite similar to those presented in Refs. [5,6,31].

\subsection{Computational costs}

This section is devoted to the comparison of the computational cost needed by the proposed method and the indicator presented in Ref. [1]. We consider the two previous examples: the flow around a cylinder (denoted by Example 1) and the lid-driven cavity (denoted by Example 2). These computational cost are given in Table 7. In this table, we give the number of matrix triangulations, $\mathrm{N} 1$, (which is the most time consuming operation in both methods) needed for each method and the corresponding CPU times. For the proposed method, the estimation of the CPU times is easy to do. For example, let us consider the flow around a cylinder (Example 1) with an initial Reynolds number equal to $39.01\left(\operatorname{Re}_{\mathrm{ini}}=39.01\right.$ in Table 7). To compute the initial guesses for the Newton method, the indicator phase requires 20 steps of the perturbation method (according to the results presented in Table 4. From this computation, the number of Newton iterations to converge (with the following required accuracy $\left\|R^{S}\right\|<10^{-4},\left\|R^{a}\right\|<10^{-8}$ and $\left.\| R^{b}\right\}<10^{-8}$ ) towards the Hopf bifurcation point is equal to 5 (see Fig. 6). The total number of triangulated matrix to determine precisely the critical values of the flow is then equal to 25 (20 for the indicator phase and 5 for the Newton method). By taking into account this number of matrix triangulations and the CPU times given in Table 5 for each step of the proposed method, the total computational cost can be estimated. Let us point out that, as the tangent matrix of the Newton method has approximatively the same size as the one of the indicator phase, we consider the same computational times for both methods.

The computational times needed to compute the bifurcation point when only indicator calculi are realized (as initially proposed in Ref. [1]) are now considered. These computational times are 


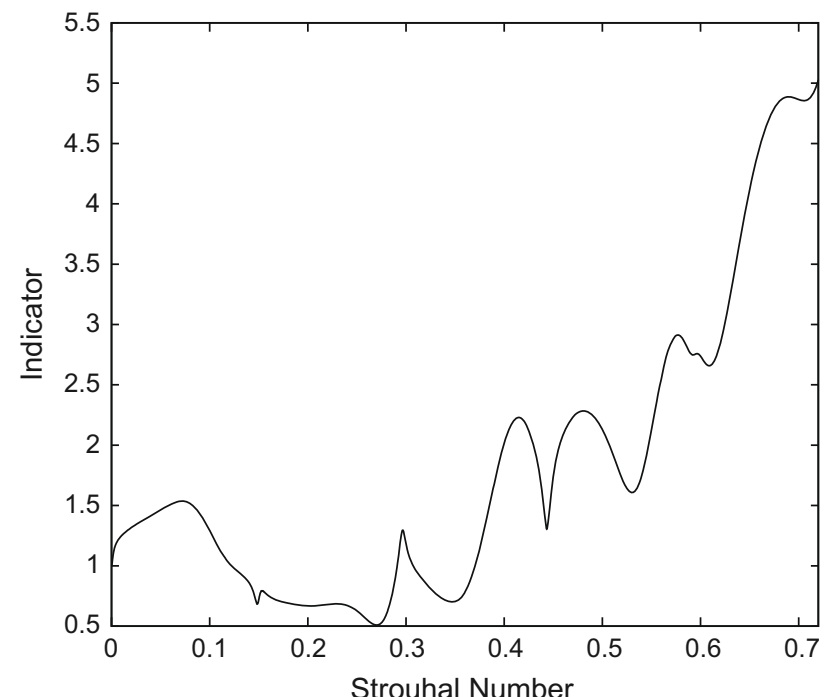

(a) $\operatorname{Re}=7257$

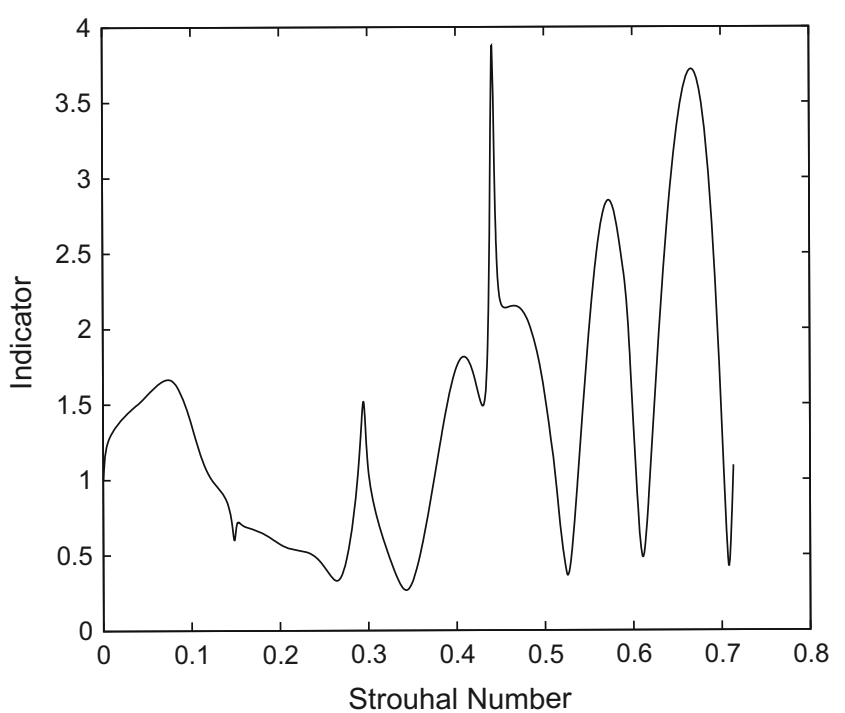

(c) $\operatorname{Re}=9296$

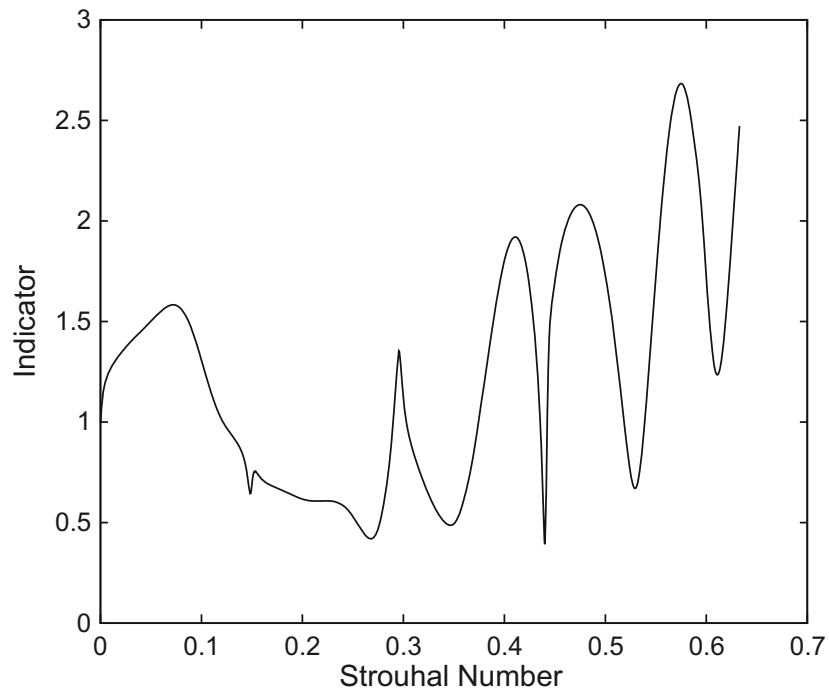

(b) $\operatorname{Re}=8089$

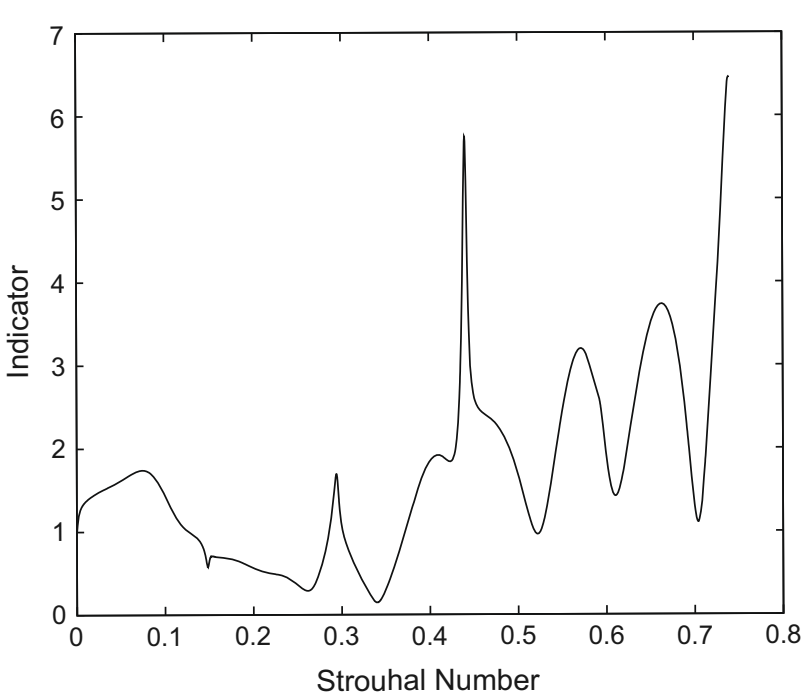

(d) $\operatorname{Re}=10147$

Fig. 7. Indicator versus angular Strouhal number. The considered example is the 2 -D lid-driven cavity with $A=1$.

Table 6

Critical parameter values obtained with several initial values. Convergence of the Newton method for the following accuracy: $\left\|R^{S}\right\|<10^{-4},\left\|R^{a}\right\|<10^{-8}$ and $\left\|R^{b}\right\|<10^{-8}$. 'NC' means no convergence of the Newton method after 20 iterations. The considered example is the lid-driven cavity, $A=1$.

\begin{tabular}{rlllrl}
\hline \multicolumn{1}{l}{$\mathrm{Re}_{\text {ini }}$} & $\mathrm{St}_{\text {ini }}$ & $\Phi_{\text {mini }}$ & Nb iterations & $\mathrm{Re}_{c}$ & $\mathrm{St}_{c}$ \\
\hline 6332.42 & 0.27 & 0.65 & $\mathrm{NC}$ & & \\
7257.45 & 0.27 & 0.508 & $\mathrm{NC}$ & & \\
7672.70 & 0.44 & 0.45 & 5 & 7890.29 & 0.44 \\
8089.12 & 0.44 & 0.39 & 4 & 7890.28 & 0.44 \\
8488.05 & 0.52 & 0.29 & 5 & 8829.59 & 0.52 \\
8888.08 & 0.52 & 0.04 & 4 & 8829.59 & 0.52 \\
9296.59 & 0.34 & 0.26 & 6 & 11286.55 & 0.33 \\
9706.36 & 0.34 & 0.206 & 7 & 11286.55 & 0.33 \\
10147.27 & 0.34 & 0.14 & 6 & 11286.55 & 0.33 \\
10589.87 & 0.33 & 0.088 & 6 & 11286.55 & 0.33 \\
\hline
\end{tabular}

more difficult to estimate than with the proposed method. As explained in Section 5.2, for Example 1, the minimum of the indicator decreases when we get closer to the critical Reynolds number and increases when we get farther from this value. This indicates that a Hopf bifurcation occurs between the tested Reynolds numbers. It means that the indicator needs at least three computations of the curve $\mathscr{G}_{\mathrm{Re}}(\Phi, \mathrm{St})$ (for three Reynolds numbers) to suggest that a Hopf bifurcation is in this range of Reynolds numbers. With these three computations, the indicator does not give a precise value for the critical values of the flow (especially for the Reynolds number). Indeed, these critical values are $\left(\operatorname{Re}_{c}=45.6, \mathrm{St}_{c}=0.137\right)$ and $\left(\mathrm{Re}_{c}=8089, \mathrm{St}_{c}=0.44\right)$ respectively for the flow around and the lid driven cavity. To compute more precise values of these critical values, supplementary indicator calculi have to be done in this range of Reynolds numbers. Finally, if with the indicator, the same accuracy as with the proposed method is required this can lead to a great number of computed indicator curves and consequently to a large computational cost. So in Table 7 , the minimum computational cost is given that permits one to prove with the indicator that a Hopf bifurcation exits in the studied range of Reynolds numbers. These computational costs are established by taking into account the results presented in Figs. 3 and 7 respectively for the Examples 1 and 2. With the results presented in Table 7, one can 


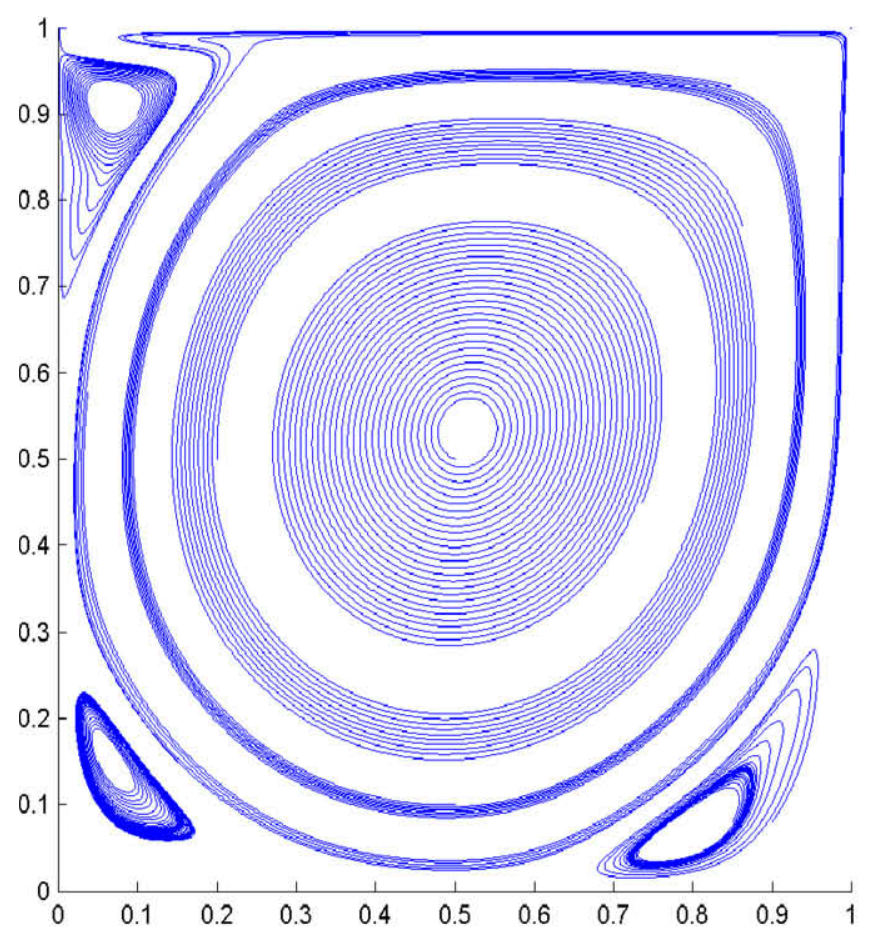

(a) Steady solution

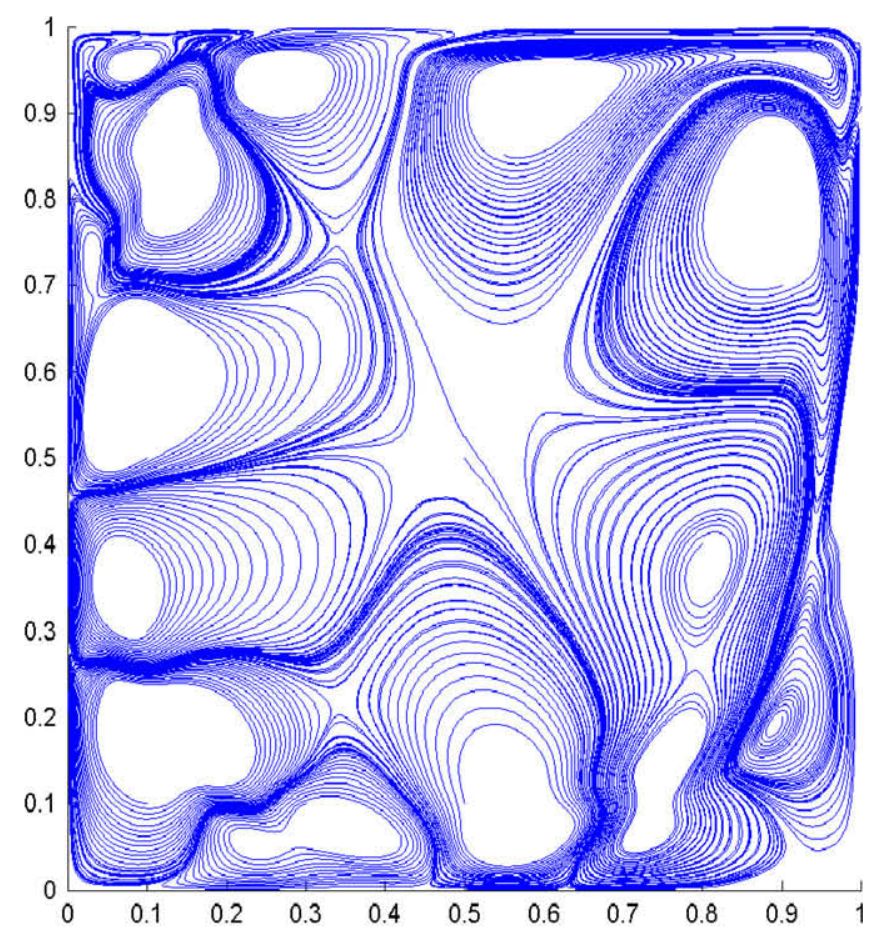

(b) Real part of the eigenvector

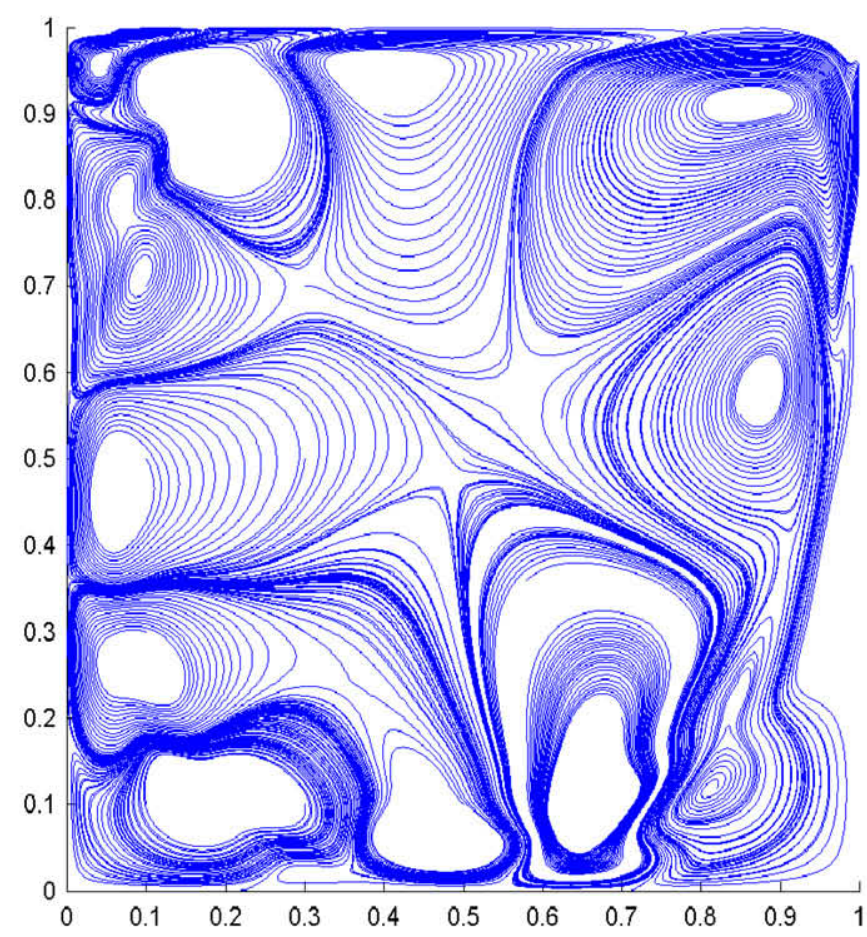

(c) Imaginary part of the eigen-

vector

Fig. 8. Streamlines for the lid-driven cavity, $A=1$, at $\operatorname{Re}=7890$. The Strouhal Number is equal to 0.44 .

clearly see that the proposed method leads to lower CPU times than the indicator proposed in Ref. [1]. So the proposed hybrid method is a real improvement of the indicator presented in Ref. [1].

\subsection{The lid-driven cavity with aspect ratio, $A=0.8$ and $A=1.5$}

We now consider the lid-driven cavity with a geometric parameter $A$ equal to 0.8 and 1.5 . The number of references concerning these two examples is relatively small compared to case $A=1$. The found critical parameters for these values of parameter $A$ are summarized in Table 9. The computations are done in the same manner as before. First the curves $\mathscr{G}_{\mathrm{Re}}(\Phi, \mathrm{St})$ are computed. Next the minimum of the indicator is automatically detected on this curve and is finally used as an initial guess for the Newton method. One considers first that case $A$ is equal to 0.8 . For this example, the following critical numbers corresponding to the first Hopf 
Table 7

Comparison of the estimated CPU time to compute the first bifurcation point with the indicator[1] and the proposed method according to the CPU time given in the Table 5 and the number of Newton iterations shown in Fig. 6 and in Table 6. N1 is the number of matrix triangulation needed and T1 is the CPU time. Examples 1 and 2 are respectively the flow around a cylinder and the lid-driven cavity with $A=1$.

\begin{tabular}{lllll}
\hline & Method & & $\mathrm{N} 1$ & $\mathrm{~T} 1(\mathrm{~s})$ \\
\hline \multirow{2}{*}{ Example 1 } & Indicator [1] & $\mathrm{Re} \in[39.01,54.92]$ & $3 \times 20$ & 610 \\
& This study & $\mathrm{Re}_{\text {ini }}=39.01$ & $20+5$ & 254 \\
& & $\operatorname{Re}_{\text {ini }}=45.6$ & $20+4$ & 244 \\
& & $\operatorname{Re}_{\text {ini }}=54.92$ & $20+5$ & 254 \\
Example 2 & Indicator [1] & $\operatorname{Re} \in[7257,8089]$ & $63+66+68$ & 13,470 \\
& This study & $\operatorname{Re}_{\text {ini }}=7672.7$ & $66+5$ & 4806 \\
& & $\operatorname{Re}_{\text {ini }}=8089$ & $68+4$ & 4885 \\
\hline
\end{tabular}

bifurcation, $\operatorname{Re}_{c}=5698$ and $\mathrm{St}_{c}=0.31$ (see Table 9), are found. In Ref. [17] (see also results in Table 8), the authors have mentioned
Table 8

Comparison of the critical parameters for the first Hopf bifurcation from the literature. The following acronyms EC and NM respectively stand for eigenvalues computation and Newton method. The considered examples are the lid-driven cavity with $A=0.8$ and $A=1.5$.

\begin{tabular}{lllll}
\hline Aspect ratio & Authors & $\mathrm{Re}_{c}$ & $\mathrm{St}_{c}$ & Method \\
\hline$A=0.8$ & Polishenko and Aidun [17] & 5225 & 0.35 & $\mathrm{NM}$ \\
& This study & 5698 & 0.31 & \\
\multirow{3}{*}{$A=1.5$} & Boppana and Gajjar [6] & 5326 & 0.34 & $\mathrm{EC}$ \\
& Polishenko and Aidun [17] & 7216 & 0.44 & $\mathrm{NM}$ \\
& Abouhamza and Pierre [31] & 5674 & 0.49 & $\mathrm{EC}$ \\
& This study & 7769 & 0.239 & \\
\hline
\end{tabular}

a critical Reynolds number equal to 5225 and an adimensional frequency close to 0.35 . These results are quite different from those found in our study. However, by comparing the streamlines of

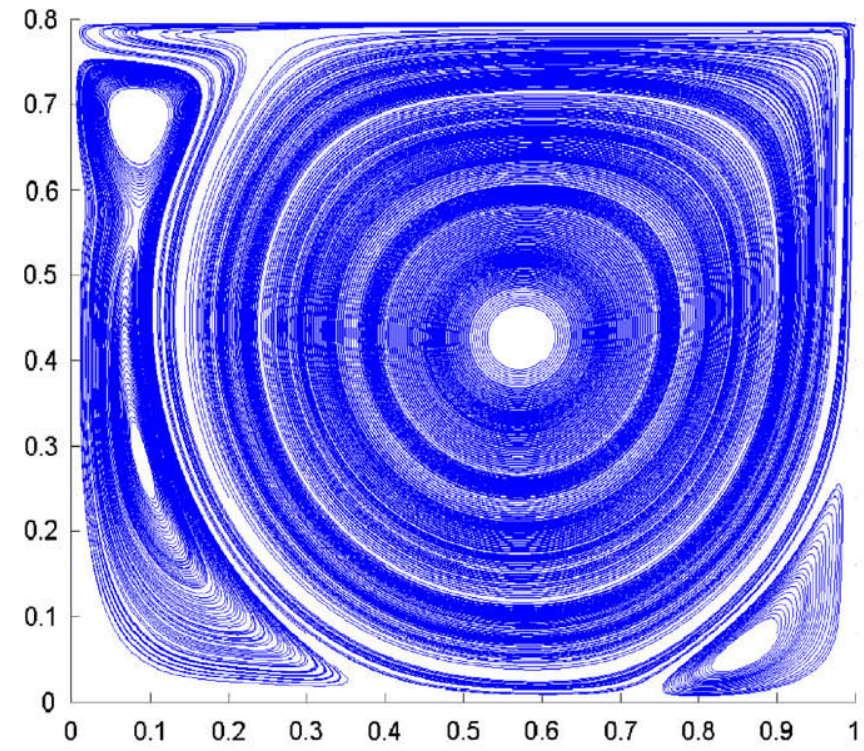

(a) Steady solution

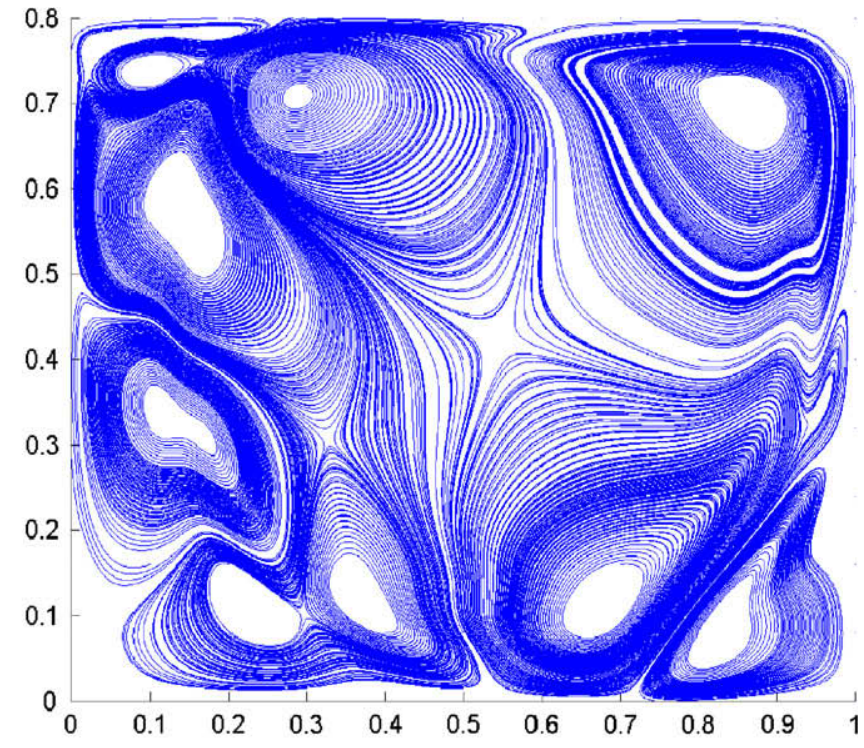

(b) Real part of the eigenvector

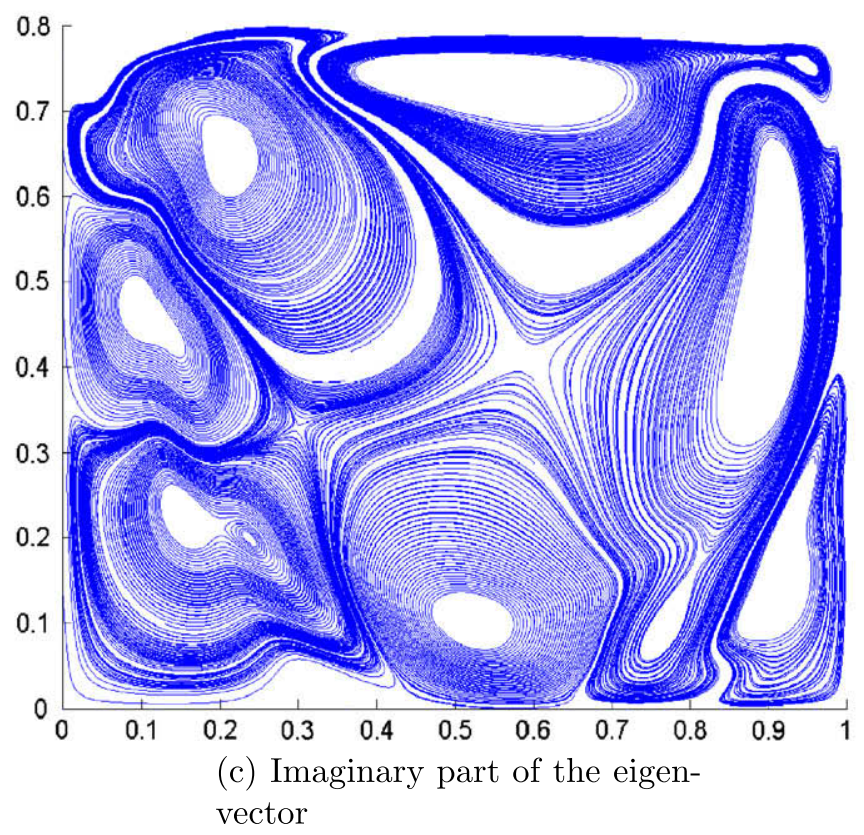

Fig. 9. Streamlines for the lid-driven cavity, $A=0.8$ plotted at $\operatorname{Re}=5698$ and $\mathrm{St}_{c}=0.31$. 
the stationary solution (at $\operatorname{Re}_{c}=5698$ ) for both real and imaginary parts of the eigenvector found in this work (see Fig. 9) and those of Ref. [17], one can clearly say that it is the same Hopf bifurcation point.

Concerning $A=1.5$, the present algorithm gives a critical Reynolds number equal to 7769 and a corresponding Strouhal number equal to 0.239 (Table 9). For this example, Polianshenko and Aidun
[17] have found $\mathrm{Re}_{c}=7216.9$ and $\mathrm{St}_{c}=0.44$ whereas Boppana and Gajjar [6] have mentioned critical Reynolds and Strouhal numbers equal respectively to 5326.9 and 0.34 (see also results in Table 8). Once more, if the stationary solution, the real and the imaginary parts of the eigenvector at this critical Reynolds number are compared (see Fig. 10) to those shown in Refs. [6,17], one comes to the same Hopf bifurcation points.

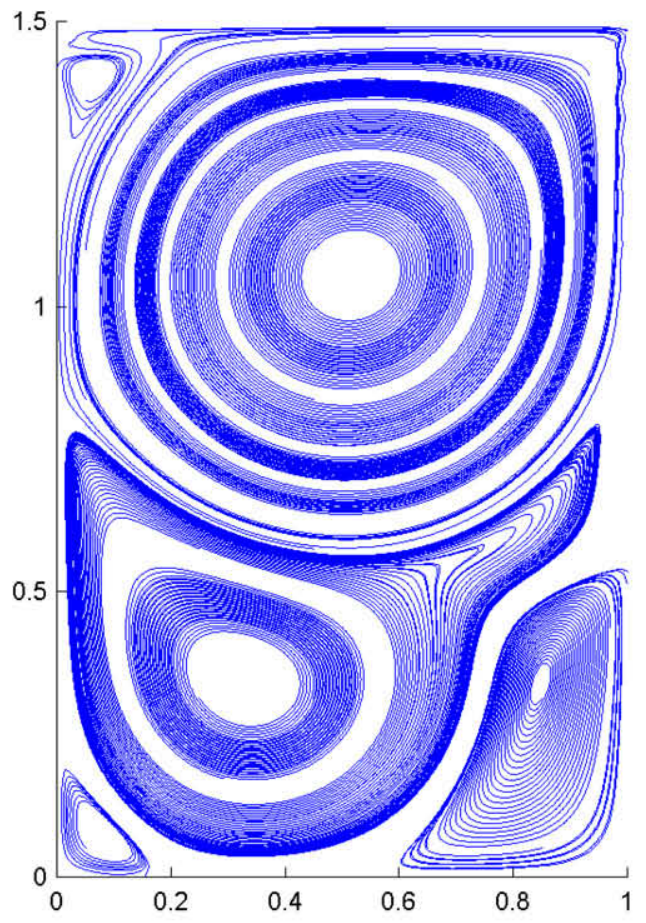

(a) Steady solution

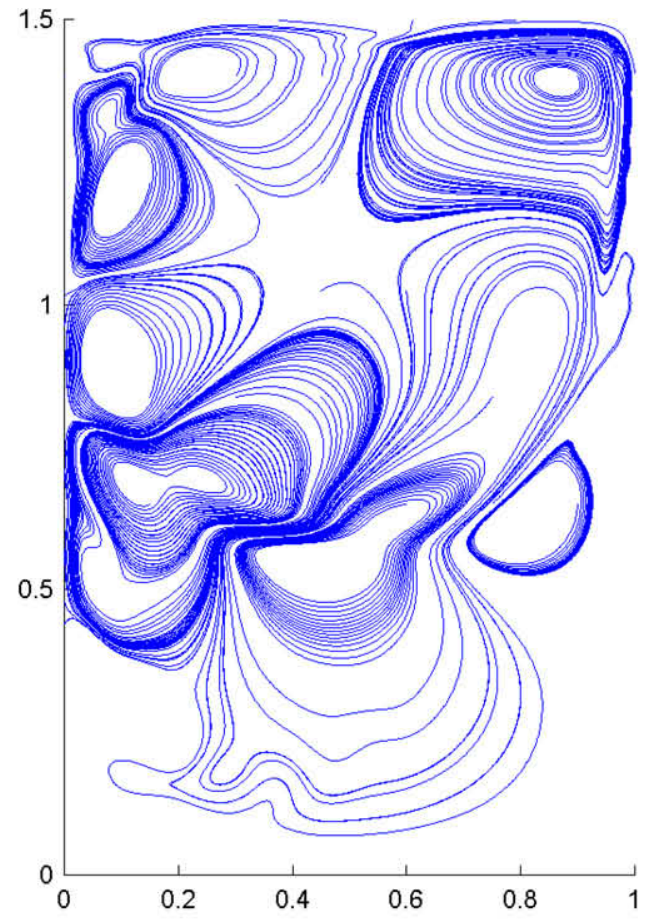

(b) Real part of the eigenvector

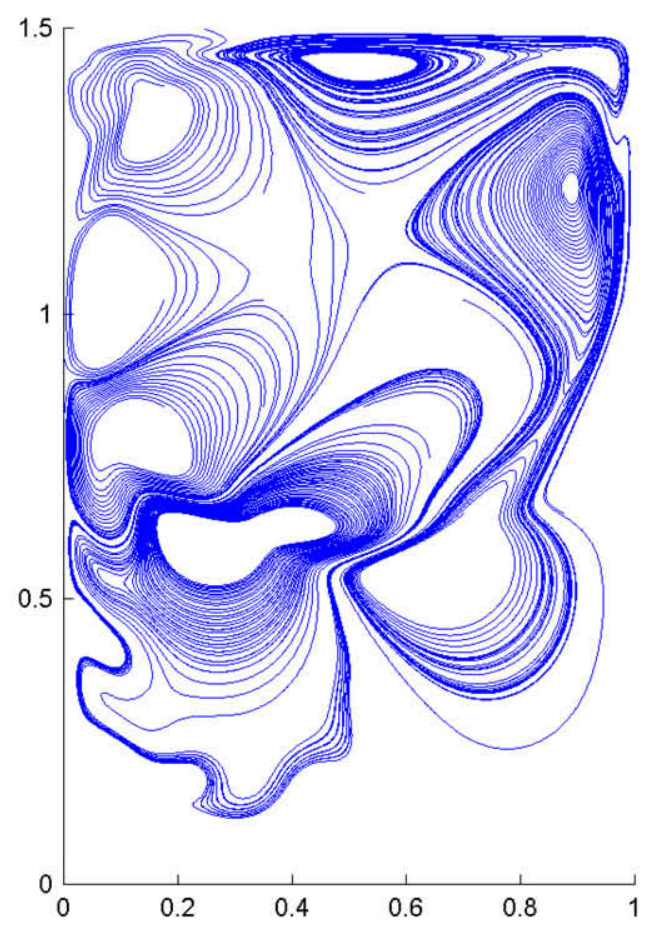

(c) imaginary part of the eigenvector

Fig. 10. Streamlines for the lid-driven cavity, $A=1.5$ plotted at $\mathrm{Re}=7769$ and $\mathrm{St}_{c}=0.24$. 
Nevertheless, Boppana and Gajjar [6] also reported a difference between the critical numbers they have found and the other works of the literature. The previous authors explain these differences by the fact that the number of computations done for these examples is relatively small (the case $A=1.5$ ). We have performed supplementary computations with other grid sizes (smaller and larger) and the critical numbers are quite similar to those reported in this paper. In conclusion, the values of the critical numbers for these two examples seem to remain an open question.

The results presented in Tables 6 and 9 show an interesting task when using the proposed algorithm to determine Hopf points. Let us consider, for example, case $A=1.5$ (Table 9). For this test, six initial values give the same bifurcation point $\left(\operatorname{Re}_{c}=7769\right.$ and $\left.\mathrm{St}_{c} \approx 0.24\right)$. These six initial guesses have approximatively the same value for the frequency, but are quite different for the Reynolds number. It shows that the bifurcation indicator can give good initial values on the stationary solution, even if the initial Reynolds numbers are far from the critical values. Moreover, results presented in Table 9 show that this is true for initial values before and after the critical Reynolds number. This is very interesting, because it means that with the indicator, we do not have to check any sign change of a function before performing the Newton method.

\subsection{Additional bifurcation points}

Another important feature concerning this work is the ability of the proposed algorithm to detect several bifurcation points. Indeed as the proposed algorithm is entirely automatic, the minimum on the curves $\mathscr{G}_{\mathrm{Re}}(\Phi, \mathrm{St})$ is computed automatically and evolves with the Reynolds number. So in this section, we present for the previous considered examples the second and the third (if it is found) Hopf bifurcation points. For the sake of simplicity, we characterize them as Hopf bifurcation points because these points are solutions of the nonlinear system (9). Few works give critical numbers for the second and following Hopf bifurcations.

We consider the lid-driven cavity with the three previous aspect ratios A. The results concerning $A=1$ are given in Table 6 and in Table 9 for $A=0.8$ and $A=1.5$. In Table 6 , one can see that the second and the third bifurcations are respectively found for $\mathrm{Re}_{c}=8829, \mathrm{St}_{c}=0.52$ and $\mathrm{Re}_{c}=11,287, \mathrm{St}_{c}=0.33$. If these results are compared with those found in the literature (see Table 10) one can remark that few results correlate well with our results or with each other. Sometimes, the critical Reynolds and frequency numbers are quite similar but the bifurcation is not the same (see results of Tiesanga et al., Table 10 , for $8875<\operatorname{Re}_{c}<9375$ and $\mathrm{St}_{c}=0.52$ for the third bifurcation point). At other times, the Reynolds number and the frequency do not correspond. Even if the physical signification of these bifurcation points can be dis-

\section{Table 9}

Critical parameter values obtained with several initial values. Convergence of the Newton method for the following accuracy: $\left\|R^{S}\right\|<10^{-4},\left\|R^{a}\right\|<10^{-8}$ and $\left\|R^{b}\right\|<10^{-8}$. 'NC' means no convergence of the Newton method after 20 iterations. The considered example is the lid-driven cavity.

\begin{tabular}{llllrl}
\hline & $\mathrm{Re}_{\text {ini }}$ & $\mathrm{St}_{\text {ini }}$ & $\mathrm{Nb}$ iterations & $\mathrm{Re}_{c}$ & $\mathrm{St}_{c}$ \\
\hline$A=0.8$ & 6023.72 & 0.31058 & 4 & 5698.57 & 0.31239 \\
& 6549.52 & 0.41938 & 4 & 6308.61 & 0.420738 \\
& 7079.31 & 0.52311 & 4 & 6707.58 & 0.524798 \\
& & & & \\
& 3077.77 & 0.00157 & $\mathrm{NC}$ & 7769.67 & 0.23941 \\
& 3658.37 & 0.26426 & 9 & 7769.67 & 0.23941 \\
& 4246.35 & 0.26141 & 11 & 7769.67 & 0.23941 \\
& 5070.06 & 0.25644 & 7 & 7769.67 & 0.23941 \\
& 5902.49 & 0.25098 & 8 & 7769.67 & 0.23941 \\
& 7025.93 & 0.24352 & 7 & 7769.67 & 0.23941 \\
& 8158.78 & 0.23768 & 6 & 10335.39 & 0.30919 \\
\hline
\end{tabular}

Table 10

Results from the literature for the second and the third Hopf bifurcation points. The considered example is the lid-driven cavity with $A=1$.

\begin{tabular}{lll}
\hline Authors & $\mathrm{Re}_{c}$ & $\mathrm{St}_{c}$ \\
\hline Tiesenga et al. [33] & $8375-8875$ & 0.6 \\
& $8875-9375$ & 0.52 \\
& $8875-9375$ & 0.7 \\
Cazemier et al. [34] & 8214 & 1.16 \\
& 10,116 & - \\
Auteri et al. [35] & 11,175 & - \\
\hline
\end{tabular}

cussed, the present method can easily and automatically give more than one bifurcation point.

Concerning the two other aspect ratios $A$, no results are reported in the literature. Thus, we just give, for information, the critical values found for the second and the third Hopf bifurcations in Table 9.

\section{Conclusion}

In this paper, we propose an algorithm to compute Hopf bifurcation points in fluid mechanics. This algorithm is based on the coupling of an indirect method (a bifurcation indicator presented in Ref. [1]) and a direct one (a nonlinear augmented system solved by the well-known Newton method). The bifurcation indicator presented in this paper is improved by using the Padé approximants which lead to less matrix triangulations and consequently saving computational times.

The bifurcation indicator gives initial values for the direct method. As these initial values are the minima values of the indicator $\Phi$, they can be computed automatically and are easily taken as initial guesses for the Newton method. Several numerical examples emanating from classical benchmarks in fluid mechanics have shown the efficiency of the proposed algorithm. Indeed, the bifurcation indicator gives initial conditions which permit a fast convergence of the Newton method. As the minimum on the curves $\mathscr{G}_{\mathrm{Re}}(\Phi, \mathrm{St})$ evolves with the Reynolds number, several bifurcation points can be easily computed. The advantage of the present algorithm is that these Hopf bifurcation points are automatically determined. In comparison with other methods, with the proposed algorithm the user does not have to check if a test function becomes null, the computation of the minima on the curves $\mathscr{G}_{\mathrm{Re}}(\Phi, \mathrm{St})$ is automatically done and finally the Newton method starts with good initial values to determine the singular points. The drawback lies in the fact that the method is based on the detection of the minimum of the curves $\mathscr{G}_{\operatorname{Re}}(\Phi, \omega)$ in a range $\left[0, \omega_{\max }\right]$. So the user has to define, from the beginning, the range of interest in $\omega$ for which a bifurcation can appear. This value of $\omega_{\max }$ is the only user parameter which seems to be difficult to define to avoid missing a bifurcation point.

Moreover, with the proposed method one can not certify, without doing a lot of computations, that the found critical values correspond to the first bifurcation point. Whereas, by computing the eigenvalues of the Jacobian matrix, this can be asserted.

The proposed method lies on the computation of at least one indicator curve, $\mathscr{G}_{\mathrm{Re}}(\Phi, \mathrm{St})$. The computation cost of this curve can be great (see the results in Table 7). This is a drawback of the proposed method if, for example, 3D flow are investigated. Nevertheless, this computational cost can be decreased by using either reduced order models for example or a linear solver which is well-adapted to repeated right-hand side problems [38] such as the ones defined in Eqs. (17) and (18). Application of these techniques to the proposed method is a subject of current studied. 
Future works also concern the use of a high order iterative method $[36,37]$ instead of the Newton one. The main objective will be to decrease the number of matrix triangulations to compute precisely the bifurcation points.

\section{Appendix A. Details on the direct method}

In this appendix, we present the direct method used in this work. During one iteration of the Newton method, one has to determine the unknown increment, $\Delta \Lambda$, which is the solution of the linearized system (12). This sytem written in a condensed form in the core of this paper is precisely defined by the following relations:

$$
\left\{\begin{array}{l}
L_{t}\left(\Delta U^{S}\right)-\Delta \lambda F=R^{S} \text { in } \Omega \\
L_{t}\left(\Delta V^{a}\right)+Q\left(V_{0}^{a}, \Delta U^{S}\right)+Q\left(\Delta U^{S}, V_{0}^{a}\right)-\omega_{0} M\left(\Delta V^{b}\right) \\
\quad-\Delta \omega M\left(V_{0}^{b}\right)=R^{a} \text { in } \Omega \\
L_{t}\left(\Delta V^{b}\right)+Q\left(V_{0}^{b}, \Delta U^{S}\right)+Q\left(\Delta U^{S}, V_{0}^{b}\right)+\omega_{0} M\left(\Delta V^{a}\right) \\
\quad+\Delta \omega M\left(V_{0}^{a}\right)=R^{b} \text { in } \Omega \\
\quad \Delta V_{u}^{a}=0 \text { on } \partial_{u} \Omega \\
\Delta V_{u}^{b}=0 \text { on } \partial_{u} \Omega
\end{array}\right.
$$

where $R^{S}, R^{a}$ and $R^{b}$ are respectively the steady residual, the real and the imaginary "dynamic" residual. $L_{t}$ is a tangent operator. The residual quantities are defined by the following expressions:

$$
\left\{\begin{array}{l}
R^{S}=-L\left(U_{0}^{S}\right)-Q\left(U_{0}^{S}, U_{0}^{S}\right)+\lambda_{0}^{S} F \text { in } \Omega \\
R^{a}=-L\left(V_{0}^{a}\right)-Q\left(V_{0}^{a}, U_{0}^{S}\right)-Q\left(U_{0}^{S}, V_{0}^{a}\right)+\omega_{0} M\left(V_{0}^{b}\right) \text { in } \Omega \\
R^{b}=-L\left(V_{0}^{b}\right)-Q\left(V_{0}^{b}, U_{0}^{S}\right)-Q\left(U_{0}^{S}, V_{0}^{b}\right)-\omega_{0} M\left(V_{0}^{a}\right) \text { in } \Omega
\end{array}\right.
$$

In the relations (25), the tangent operator $L_{t}$ has been introduced. This operator is defined by:

$L_{t}(\bullet)=L(\bullet)+Q\left(\bullet, U_{0}^{S}\right)+Q\left(U_{0}^{S}, \bullet\right)$

\section{Appendix B. Range of validity of asymptotic expansion and Padé approximants}

Once the polynomial approximation (15) is computed by solving the linear sytems (17) and (18), one have to define its range of validity. This forwards defining a new starting point inside this domain of validity and finally by reapplying the perturbation method from this point to determine the following part of the solution.

In the case of polynomial approximation, a very simple criterion has been proposed in Ref. [13]. The idea is to require that the difference between two consecutive order solutions is smaller than a chosen small parameter $\epsilon$ (typically $10^{-3} \leqslant \epsilon \leqslant 10^{-6}$ ):

$\frac{\left\|V^{a}(N)-V^{a}(N-1)\right\|}{\left\|V^{a}(N)-V_{0}^{a}\right\|}=\frac{\left\|\hat{\omega}^{N} V_{N}^{a}\right\|}{\left\|\hat{\omega} V_{1}^{a}+\cdots+\hat{\omega}^{N} V_{N}^{a}\right\|} \leqslant \epsilon$

where $V^{a}(N)$ is the series expansions (15) computed at the order of truncature $p=N$. By considering that the denominator of the previous relation can be estimated by $\left\|\hat{\omega} V_{1}^{a}\right\|$, this leads to the following definition of the maximum value, $\hat{\omega}_{\max }$ Poly, of the perturbation parameter $\hat{\omega}$ :

$\hat{\omega}_{\max \text { Poly }}=\left(\epsilon \frac{\left\|V_{1}^{a}\right\|}{\left\|V_{N}^{a}\right\|}\right)^{1 /(N-1)}$

In Ref. [22], the authors have extended the criterion (28) to the Padé approximants (19). By requiring that the difference between two rational solutions (19) at consecutive orders remains smaller at the end of the step than a chosen parameter, $\delta$, the maximum value,

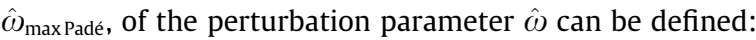

$\delta=\frac{\left\|V_{\text {Padé, } N}^{a}\left(\hat{\omega}_{\text {max Padé }}\right)-V_{\text {Padé },(N-1)}^{a}\left(\hat{\omega}_{\text {max Padé }}\right)\right\|}{\left\|V_{\text {Padé }, N}^{a}\left(\hat{\omega}_{\max \text { Padé }}\right)-V_{0}^{a}\right\|}$

One can remark that with the previous relation, we do not have an explicit relation of the validity range of the Pade approximants, contrariwise to polynomial approximations with the relation (29). In fact, the maximum value of the perturbation parameter, $\hat{\omega}_{\max }$ Padé, has to be evaluated with expression (30) in the range $\left[\hat{\omega}_{\max \text { Poly }}, r_{1}\right]$, where $r_{1}$ designates the first pole of the Padé approximants.

Finally, the relations (29) and (30) permit defining an automatic continuation procedure by successively reapplying the perturbation method in a step-by-step manner. Let us remark that the two parameters $\epsilon$ and $\delta$ play the same role: they govern the step length and the accuracy, respectively of the asymptotic expansions and the Padé approximants.

\section{References}

[1] Cadou JM, Potier-Ferry M, Cochelin B. A numerical method for the computation of bifurcation points in fluid mechanics. Eur J Mech B: Fluids 2006;25:234-54.

[2] Jackson CP. A finite-element study of the onset of vortex shedding in flow past variously shaped body. J Fluid Mech 1987;182:23-45

[3] Jepson AD. Numerical Hopf bifurcation. Thesis, California Institute of technology; 1981.

[4] Hassard B, Kazarinoff N, Wan Y-H. Theory and applications of Hopf bifurcation. Cambridge University Press; 1981.

[5] Fortin A, Jardak M, Gervais JJ, Pierre R. Localization of Hopf bifurcations in fluid flow problems. Int J Numer Methods Fluids 1997;24:1185-210.

[6] Boppana VBL, Gajjar JSB. Global flow instability in a lid-driven cavity. Int J Numer Methods Fluids 2010;62(8):827-53.

[7] Nayar N, Ortega JM. Computation of selected eigenvalues of generalized eigenvalue problems. J Comput Phys 1993;108:8-14.

[8] Boutyour EH, Zahrouni H, Potier-Ferry M, Boudi M. Bifurcation points and bifurcated branches by an asymptotic numerical method and Padé approximants. Int J Numer Methods Eng 2004;60:1987-2012.

[9] Vannucci P, Cochelin B, Damil N, Potier-Ferry M. An asymptotic-numerical method to compute bifurcating branches. Int J Numer Methods Eng 1998;41:1365-89.

[10] Allery C, Cadou JM, Hamdouni A, Razafindralandy D. Application of the asymptotic numerical method to the Coanda effect study. Revue Européenne des Eléments Finis 2004;13:57-77.

[11] Tri A. Méthodes Asymptotiques-Numériques pour les fluides visqueux incompressibles et la détection de la bifurcation de Hopf. Thesis, Université de Metz, France; 1996.

[12] Bensaadi MEH. Méthode Asymptotique-Numérique pour le calcul de bifurcations de Hopf et de solutions périodiques. Thesis, Université de Metz, France; 1995.

[13] Cochelin B. A path-following technique via an asymptotic-numerical method. Comput Struct 1994;53(5):1181-92.

[14] Griewank A, Reddien G. The computation of Hopf points by a direct method. IMA J Numer Anal 1983;3:295-303.

[15] Roose D. An algorithm for the computation of Hopf bifurcation points in comparison with other methods. J Comput Appl Math 1985;12-13:517-29.

[16] Hussein A, Chen K. On efficient methods for detecting Hopf bifurcation with applications to power system instability prediction. Int J Bifurcat Chaos 2003;13(5):1247-62.

[17] Poliashenko M, Aidun CK. A direct method for computation of simple bifurcations. J Comput Phys 1995;121:246-60.

[18] Cadou JM, Cochelin B, Damil N, Potier-Ferry M. Asymptotic numerical method for stationary Navier-Stokes equations and with Petrov-Galerkin formulation. Int J Numer Methods Eng 2001;50:825-45.

[19] Baker GA, Graves-Morris P. Padé approximants, encyclopedia of mathematics and its applications. Second ed. Cambridge: Cambridge University Press; 1996.

[20] Braikat B, Damil N, Potier-Ferry M. Méthodes asymptotiques numériques pour la plasticité. Revue Européenne des Eléments Finis 1997;6:337-57.

[21] Najah A, Cochelin B, Damil N, Potier-Ferry M. A critical review of asymptotic numerical method. Arch Comput Methods Eng 1998;5(1):3-22.

[22] Elhage-Hussein A, Potier-Ferry M, Damil N. A numerical continuation method based on Padé approximants. Int J Solids Struct 2000;37:6981-7001.

[23] Zienkiewicz OC, Taylor RL. The finite element method. 4th ed., vol. 2. McGrawHill Book Company; 1991.

[24] Potier-Ferry M, Cadou JM. Basic ANM algorithms for path following problems. Revue Européenne des Eléments finis 2004:13:9-32.

[25] Cossu C, Morino L. A vorticity-only formulation and a low-order asymptotic expansion solution near Hopf bifurcation. Comput Mech 1997;20:229-41. 
[26] Kumar B, Mittal S. Prediction of the critical Reynolds number for flow past a circular cylinder. Comput Methods Appl Eng 2006;195:6046-58.

[27] Ding Y, Kawahara M. Three-dimensional linear stability analysis of incompressible viscous flows using the finite element method. Int J Numer Methods Fluids 1999;31:451-79.

[28] Morzynski M, Afanasiev K, Thiele F. Solution of the eigenvalue problems resulting from global non-parallel flow stability analysis. Comput Methods Appl Mech Eng 1999;169:161-76.

[29] Bruneau CH, Saad M. The 2D lid-driven cavity problem revisited. Comput Fluids 2006;35:326-48.

[30] Peng YF, Shiau YH, Hwang RR. Transition in 2-D lid-driven cavity flow. Comput Fluids 2003;32:337-52

[31] Abouhamza A, Pierre R. A neutral stability curve for incompressible flows in a rectangular driven cavity. Math Comput Model 2003;38:141-57.

[32] Gervais JJ, Lemelin D, Pierre R. Some experiments with stability analysis of discrete incompressible flows in the lid-driven cavity. Int J Numer Methods Fluids 1997;24:477-92.
[33] Tiesinga G, Wubs FW, Veldman AEP. Bifurcation analysis of incompressible flow in a driven cavity by the Newton-Picard method. J Comput Appl Math 2002;140:751-72

[34] Cazemier W, Verstappen RWCP, Veldman AEP. Proper orthogonal decomposition and low-dimensional models for driven cavity flows. Phys Fluids 1998;10-7:1685-99.

[35] Auteri F, Parolini N, Quartapelle L. Numerical investigation on the stability of singular driven cavity flow. J Comput Phys 2002;183:1-25.

[36] Cadou J-M, Damil N, Potier-Ferry M, Braikat B. Projection techniques to improve high-order iterative corrector. Finite Elem Anal Des 2004;41:285309.

[37] Mallil E, Lahmam H, Damil N, Potier-Ferry M. An iterative process based on homotopy and perturbation techniques. Comput Methods Appl Mech Eng 2000;190(13-14):1845-58.

[38] Cadou JM, Potier-Ferry M. A solver combining reduced basis and convergence acceleration with applications to non-linear elasticity. Commun Numer Methods Eng, doi:10.1002/cnm.1246. 